\title{
The brown dwarf population in the Chamaeleon I cloud ${ }^{\star}$
}

\author{
B. López Martí ${ }^{1,2}$, J. Eislöffel ${ }^{2}$, A. Scholz ${ }^{2}$, and R. Mundt ${ }^{3}$ \\ 1 Observatori Astronòmic de la Universitat de València, Edifici d'Instituts d'Investigació, Polígon La Coma, \\ 46980 Paterna, Spain \\ 2 Thüringer Landessternwarte, Sternwarte 5, 07778 Tautenburg, Germany \\ e-mail: jochen@tls-tautenburg.de; scholz@tls-tautenburg.de \\ 3 Max-Planck-Institut für Astronomie, Königstuhl 17, 69117 Heidelberg, Germany \\ e-mail: mundt@mpia-hd.mpg.de
}

Received 16 April 2003 / Accepted 19 November 2003

\begin{abstract}
We present the results of a multiband survey for brown dwarfs in the Chamaeleon I dark cloud with the Wide Field Imager (WFI) camera at the ESO/MPG 2.2-m telescope on La Silla (Chile). The survey has revealed a substantial population of brown dwarfs in this southern star-forming region. Candidates were selected from $R, I$ and $\mathrm{H} \alpha$ imaging observations. We also observed in two medium-band filters, M 855 and M 915, for spectral type determination. The former filter covers a wavelength range containing spectral features characteristic of M-dwarfs, while the latter lies in a relatively featureless wavelength region for these late-type objects. A correlation was found between spectral type and (M 855-M 915) colour index for mid- to late M-type objects and early L-type dwarfs. With this method, we identify most of our object candidates as being of spectral type M 5 or later. Our results show that there is no strong drop in the number of objects for the latest spectral types, hence brown dwarfs may be as abundant as low-mass stars in this region. Also, both kind of objects have a similar spatial distribution. We derive an index $\alpha=0.6 \pm 0.1$ of the mass function in this region of dispersed star formation, in good agreement with the values obtained in other star forming regions and young clusters. Some of the brown dwarfs have strong $\mathrm{H} \alpha$ emission, suggesting mass accretion. For objects with published infrared photometry, we find that strong $\mathrm{H} \alpha$ emission is related to a mid-infrared excess, indicative of the existence of a circumstellar disk.
\end{abstract}

Key words. stars: low-mass, brown dwarfs - stars: pre-main sequence - stars: formation stars: luminosity function, mass function - stars: circumstellar matter

\section{Introduction}

Our understanding of substellar objects has considerably increased in the last years. Recent surveys have revealed important populations of brown dwarfs in young open clusters and star forming regions (e.g. Bouvier et al. 1998; Béjar et al. 1999; Wilking et al. 1999; Lucas \& Roche 2000; Barrado y Navascués et al. 2001; Zapatero Osorio et al. 2002b; Lamm et al. 2004). Still, little is known about the properties of very young substellar objects (ages $<10^{8} \mathrm{yr}$ ).

An open question is the way brown dwarfs form. Although it is commonly accepted that they originate, like low-mass stars, from the gravitational collapse of molecular cloud cores, their formation process is still poorly understood. The number of observed brown dwarfs seems to vary from one region to another. For instance, some Orion clusters are rich in substellar objects (Lucas \& Roche 2000; Béjar et al. 1999), while

Send offprint requests to: B. López Martí,

e-mail: belen.lopez-marti@uv.es

* Based on observations collected at the European Southern Observatory, La Silla, Chile. in Taurus only a few brown dwarfs are known so far (Briceño et al. 1998; Martín et al. 2001). Hence, environmental conditions may play an important role in the formation of brown dwarfs. Stellar winds from a nearby hot massive star could evaporate the envelopes of accreting cores before they reach a mass above the hydrogen burning limit. Another possibility is that brown dwarfs are stellar embryos whose growth stopped after they were dynamically ejected from small stellar systems (Reipurth \& Clarke 2001; Bate et al. 2003). Brown dwarfs may also form like planets, in accretion disks around stars (e.g. Pickett et al. 2000). In this case, most of them would have been ejected from their parental systems to account for the so-called "brown dwarf desert" (e.g. Sterzik \& Durisen 1999). Another way to explain the lack of brown dwarf companions in wide orbits around stars is that they have migrated inwards due to their high mass (compared to planets). Eventually, they could remain in closer orbits, or continue their migration until they merge with the central star (Armitage \& Bonnell 2002).

The Chamaeleon I cloud is a favourable region for the study of the formation of substellar objects, because of its proximity ( $160 \mathrm{pc}$, Whittet et al. 1997) and high galactic 
latitude $\left(b \approx-16^{\circ}\right)$, which decreases the effects of contamination by other late-type stars in the Galaxy. It is the largest of the three dark clouds in the Chamaeleon complex (Gregorio Hetem et al. 1989), and apparently also the oldest. The cloud contains more than 150 known young stars (e.g. Gauvin \& Strom 1992; Feigelson et al. 1993; Appenzeller \& Mundt 1989) with a mean age of about $3 \mathrm{Myr}$, most of them clustered in two cloud cores containing two intermediate mass stars, HD 97048 and HD 97300. Chamaeleon I has also been surveyed for brown dwarfs: Neuhäuser \& Comerón (1998) reported the discovery of the very first X-ray emitting brown dwarf, $\mathrm{ChaH} \alpha 1$, in Chamaeleon I. In subsequent observations, another twelve bona-fide brown dwarfs and brown dwarf candidates (with masses between about 0.08 and $0.03 M_{\odot}$ ) were found in a small region around HD 97048 (Comerón et al. 1999; Comerón et al. 2000; hereafter CRN99 and CNK00, respectively).

This paper presents a new large and deep survey for brown dwarfs in the Chamaeleon I cloud. In Sect. 2 we describe the reduction and photometry of our data. Our candidate selection criteria follow in Sect. 3. A method for the spectral type identification of $\mathrm{M}$ and early L-dwarfs from direct imaging is presented. We discuss our object classification, and compare our identifications with available near- and mid-infrared data. We then proceed to study the possible binarity of the lowmass objects in Chamaeleon I (Sect. 5), their spatial distribution (Sect. 4), their accretion indicators (Sect. 6) and their mass function (Sect. 7). The implications of our results for the proposed brown dwarf formation models are discussed in Sect. 8 . We summarize our conclusions in Sect. 9.

\section{Observations and data processing}

\subsection{WFI observations}

The Wide Field Imager (WFI) is a focal reducer-type camera mounted at the Cassegrain focus of the 2.2-m ESO/MPG telescope at La Silla (Baade et al. 1998). It consists of a mosaic of eight $2 \times 4 \mathrm{~K} \mathrm{CCD}$ detectors with narrow interchip gaps (the filling factor is 95.9\%). The detector has a field of view of about $34^{\prime} \times 33^{\prime}$ and a pixel size of $0{ }^{\prime} 24$.

In Chamaeleon I, our survey consists of four WFI fields, covering an area of about $1.2 \square^{\circ}$ (see Fig. 1). Two of these fields contain the intermediate mass stars HD 97048 and HD 97300, respectively, around which most of the young low-mass stellar population is clustered. The field previously surveyed for brown dwarfs (CRN99; CNK00) covered a region around HD 97048 that represents about $15 \%$ of the total area of our Chal-4 field. The other two fields lie in regions where no intermediate mass stars are present: ChaI-5 to the south of ChaI-4, and ChaI- 6 in the region between ChaI-4 and ChaI-7. We did not dithered between the fields, so some objects might be missed because they fall in an interchip gap.

The observations were carried out on three different nights in May/June 1999. We observed in the broad-band filters $R$ and $I$, for the purpose of candidate selection, and in a $\mathrm{H} \alpha$ filter, to test the youth of our objects. Because we wanted to attempt a photometric spectral classification (see Sect. 3.2), we

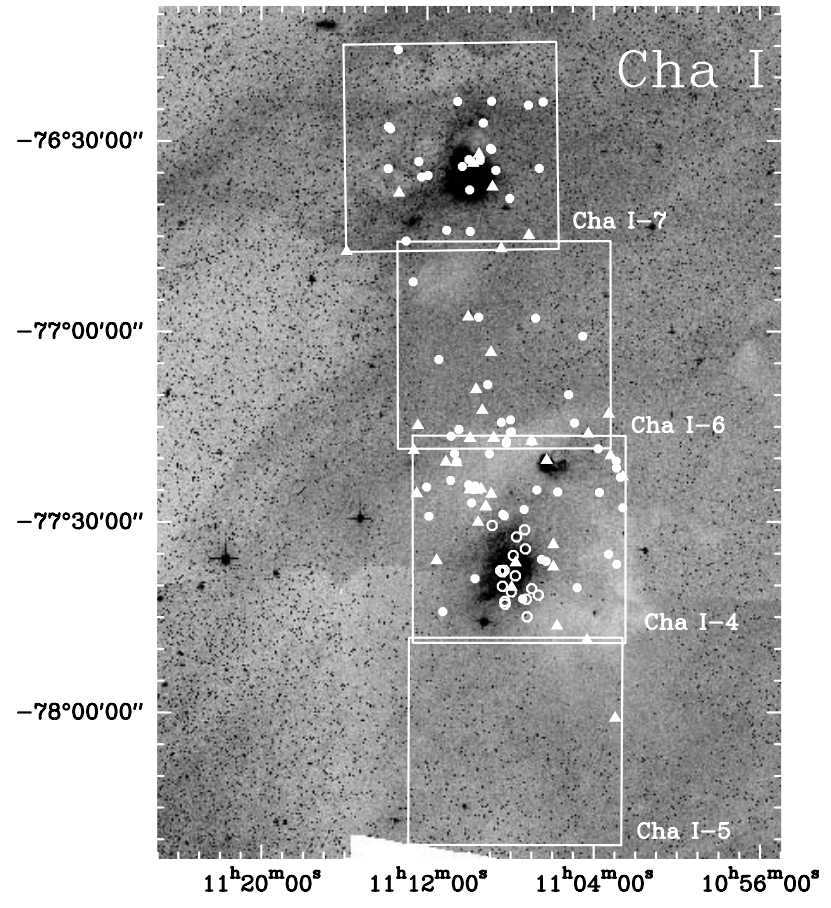

Fig. 1. DSS image of the Chamaeleon I cloud showing the location of our four WFI fields, ChaI-4 through 7. The open circles indicate the positions of the objects studied by CRN99 and CNK00; the filled circles and triangles, of our candidates with and without $\mathrm{H} \alpha$ emission, respectively.

also observed in two medium-band filters, $855 / 20$ and 915/28 (hereafter M 855 and M915, respectively), centred inside and outside $\mathrm{TiO}$ and $\mathrm{VO}$ absorption bands. These bands get deeper with later M-subspectral type, but disappear in K- and L-objects.

For each field and filter, we took three exposures with different exposure times, in order to prevent the brightest $\mathrm{T}$ Tauri stars (TTS) from being saturated. For the $\mathrm{H} \alpha$ filter only two different exposure times were used. For the field ChaI-7 this observing programme could not be carried out completely due to technical problems, and in this case we only have images with short and intermediate exposure times in the $R$ and $I$ filters. The average seeing varied from $\sim 1^{\prime \prime}$ for the field ChaI-7 to $\sim 1$.'7 for ChaI-5. The log of our observations is shown in Table 1.

Bias exposures and skyflats were taken in each filter. For the broad-band photometry calibration, several Landolt fields (Landolt 1992) were observed in all nights. We also observed a list of stars with known late spectral type (between K 7 and L 3) to use them as standards for the spectral type calibration (see Sect. 3.2).

\subsection{Data reduction and photometry}

The image processing (data reduction and photometry) was run with the MSCRED and CCDRED packages in the IRAF

\footnotetext{
1 The transmission of these filters was remeasured on December 16th 1999, and they were re-named 856/14 and 914/27, respectively (see the WFI web site for details). However, we will keep the old nomenclature throughout this paper.
} 
Table 1. Log of WFI observations in Chamaeleon I.

\begin{tabular}{llcc}
\hline \hline Date & Field & Filter & Exp. Times (s) \\
\hline 28 May 1999 & ChaI-4 & Rc/162 & $600 / 60 / 5$ \\
28 May 1999 & ChaI-4 & Ic/lwp & $600 / 30 / 5$ \\
28 May 1999 & ChaI-4 & H $\alpha / 7$ & $600 / 15$ \\
28 May 1999 & ChaI-4 & $855 / 20$ & $600 / 300 / 16$ \\
28 May 1999 & ChaI-4 & $915 / 28$ & $600 / 100 / 8$ \\
\hline 28 May 1999 & ChaI-6 & Rc/162 & $600 / 60 / 5$ \\
28 May 1999 & ChaI-6 & Ic/lwp & $600 / 30 / 5$ \\
28 May 1999 & ChaI-6 & H $\alpha / 7$ & $600 / 15$ \\
28 May 1999 & ChaI-6 & $855 / 20$ & $600 / 300 / 16$ \\
28 May 1999 & ChaI-6 & $915 / 28$ & $600 / 100 / 8$ \\
\hline 30 May 1999 & ChaI-7 & Rc/162 & $60 / 5$ \\
30 May 1999 & ChaI-7 & Ic/lwp & $30 / 5$ \\
30 May 1999 & ChaI-7 & H $\alpha / 7$ & $600 / 15$ \\
30 May 1999 & ChaI-7 & $855 / 20$ & $600 / 300 / 16$ \\
30 May 1999 & ChaI-7 & $915 / 28$ & $600 / 100 / 8$ \\
\hline 3 Jun. 1999 & ChaI-5 & Rc/162 & $600 / 60 / 5$ \\
3 Jun. 1999 & ChaI-5 & Ic/lwp & $600 / 30 / 5$ \\
3 Jun. 1999 & ChaI-5 & H $\alpha / 7$ & $600 / 15$ \\
3 Jun. 1999 & ChaI-5 & $855 / 20$ & $600 / 300 / 16$ \\
3 Jun. 1999 & ChaI-5 & $915 / 28$ & $600 / 100 / 8$ \\
\hline
\end{tabular}

environment ${ }^{2}$. Due to the large field of view, a standard reduction of WFI images (bias subtraction and flat field division) is not enough to obtain good photometry results: first, because the illumination of the sky flat fields and the science images is not equivalent, there remains an illumination gradient in the background of our object exposures after the flatfielding. To correct for this effect, the image has to be divided through this gradient or illumination mask. Second, due to the long exposure times, a fringing pattern appears. This effect is especially strong in the $I$ band. To correct for it, one has to subtract an individually scaled fringe mask from each science image. The fringes cannot be completely removed, however, because their intensity varies from one chip to the other and even between different areas of the same chip. On the other hand, in the $R$ and $\mathrm{H} \alpha$ images, as well as in most of the images with short exposure times, the fringing effect is negligible, and therefore we only corrected for the illumination gradient in those cases. Both the illumination and the fringe masks were created by combination of the science exposures.

For the astrometry, we followed the standard method proposed in Francisco Valdés' Guide to the NOAO Mosaic Data Handling Software ${ }^{3}$. Stars from the Guide Star Catalogue were selected as reference for the astrometric calibration

\footnotetext{
${ }^{2}$ IRAF is distributed by the National Optical Astronomy Observatory (NOAO), which isoperated by the Association of Universities for Research in Astronomy, Inc. under contract to the National Science Foundation.

3 This guide is available from: http://iraf.noao.edu/scripts/irafhelp?mscguide
}

(Morrison et al. 2001 and references therein). The astrometric error is in general not larger than $1^{\prime \prime}$.

Object search was then performed on each individual chip of the long exposures using SExtractor (Bertin \& Arnouts 1996). After several tests, we concluded that this software worked better than DAOFIND, leading to fewer spurious detections.

For the photometry, the DAOPHOT package was used (Stetson 1987). Since the DAOPHOT tasks only work on individual CCDs, a special IRAF procedure was written for the WFI images. We first performed aperture photometry with the PHOT task, and then PSF-photometry using the PSF and ALLSTAR tasks, regardless of the crowdedness of the field. In this way we intended to measure the faintest components of multiple systems. This did not prevent some very faint objects to be merged with their much brighter neighbours, however, although we allowed only for a very small merging radius. This problem affects to objects up to separations of about 4 " depending on the relative brightness of the objects. Given that the fraction of faint objects lost due to this problem is small (less than 3\%) in such an uncrowded region, we decided not to further consider these objects in our study. Only in a few interesting cases (see Sect. 5), aperture photometry was performed on the subtracted images.

On most of the short exposures only aperture photometry was performed, because of the difficulty of fitting a suitable PSF. This was also the case of the standard stars (exposure times of 5-10s).

\subsection{Photometric calibration}

The two broad-band filters were calibrated with Landolt (1992) standard stars. Absolute magnitudes were computed using the relations:

$$
\begin{aligned}
& R=r+B_{1}+B_{2} \cdot X_{R}+B_{3} \cdot(r-i) \\
& I=i+A_{1}+A_{2} \cdot X_{I}+A_{3} \cdot(r-i)
\end{aligned}
$$

where $r$ and $i$ are the instrumental magnitudes, $X_{R}$ and $X_{I}$ denote the measured airmasses, and $A_{k}$ and $B_{k}$ are the coefficients obtained from a linear fit using the standard instrumental and absolute magnitudes.

In the first two nights (May 28th and May 30th), five Landolt fields containing more than 60 standard stars were observed. Their airmasses range from 1.15 to 2.90 . The fits for these nights have a standard deviation of about $5 \%$. In the third night (June 3rd), only two Landolt fields were taken, although one of them was observed twice at different airmass. The number of stars used for the fit (the stars in the repeated field were used twice) was 29 , with airmasses between 1.14 and 1.95. The standard deviation is about 6\%.

The resulting coefficients are somewhat different for each night (see Table 2). In particular the colour term for the $I$ band, $A_{3}$, is relatively high. This is not surprising, because the shape of the transmission curve for this filter differs notably from a standard Cousins $I$ filter, especially at shorter wavelengths. On the other hand, the $R$ filter shows a good match with a Cousins 
Table 2. Calibration coefficients for the WFI $I$ and $R$ filters.

\begin{tabular}{lcccccc}
\hline \hline Night & $A_{1}$ & $A_{2}$ & $A_{3}$ & $B_{1}$ & $B_{2}$ & $B_{3}$ \\
\hline May 28th & -1.38 & -0.13 & 0.23 & -0.21 & -0.24 & 0.00 \\
May 30th & -1.47 & -0.03 & 0.25 & -0.58 & 0.00 & 0.00 \\
June 3rd & -1.19 & -0.19 & 0.32 & -0.18 & -0.23 & 0.00 \\
\hline
\end{tabular}

$R$ filter, and in this case the colour term is so small that it can be set to zero (see the WFI User Manual for details).

The main error source in this calibration is the fact that it comes from a global fit for all the CCD chips. Recently, Alcalá et al. (2002) showed that, for the WFI, chip-to-chip photometric variations can be as high as $3 \%$ in the broad-band filters and $5 \%$ in medium-band filters, because of different zero-points for the individual CCDs. With no doubt, a better result could be obtained by fitting the stars in each frame separately. This was not possible in our case, however, because some CCD chips contained few or no standard stars (especially those in the corners). Future observations should take several exposures dithering the fields, so that enough stars are observed on each CCD.

We also note that the Landolt stars used for the photometric calibration span a range in $(R-I)$ colours of about -0.1 to 1.5 , while our objects of interest have colours in the range 0.5 to 4 (see Sect. 3.1). Hence, our colours may not be exactly in the Cousins system, especially for the reddest objects with the largest colour terms. This is a general problem that affects all studies of very red objects, as long as no set of very red standard stars is available. For the WFI colours, comparison with the predictions of the Lyon models (Baraffe et al. 1998; Chabrier et al. 2000) shows deviations of up to 0.5 mag for very red objects $(R-I=2.5)$, while there is good agreement for $R-I=1.5$ (see also Eislöffel et al., in prep.).

Apart from this, our photometry shows a systematic error of unknown origin: using the objects present in the images with different exposure times to estimate the error in our photometry, we found a systematic offset between them. In general, the resulting luminosities are brighter by a constant amount (typically between 0 . and $0.06 \mathrm{mag}$, though as high as $\sim 0.1 \mathrm{mag}$ in the worst case) for longer exposure times. This effect is reproduced with various photometry packages and with different parameters. It is present already in the raw data (i.e., previous to the reduction), and does not show any obvious correlation with the observed field, the filter used or the measured extinction. Observing conditions are not likely to have caused it, because the images were taken one after another, and the effect is always in the same direction for all images in all photometric nights. Extensive tests by the ESO staff have verified that the effect is not caused by a WFI shutter malfunction leading to systematically short or variable integration times. It seems also improbable that it is caused by detector saturation, because the objects appear brighter, not fainter, with longer exposure times.

Therefore, to homogenize our photometry, we have determined the offsets between the three different integration times, and have shifted the short and long integration times to the system of the medium integration. That way, we should have minimized this systematic error, since the medium integration times for our objects are not very different from those of the standard stars. We did not choose the shortest exposures as reference because they contained only a few objects which could be used to estimate the exposure time offsets.

Since our observed fields in Chamaeleon I are slightly overlapping, we checked for double detections of objects. We found that the photometry results in the overlapping region between two fields are consistent within the estimated errors. Comparison with DENIS I photometry for previously known objects in our field ChaI-4 (Neuhäuser \& Comerón 1999) gives differences of about 0.2 mag. Hence, our results are marginally consistent with the DENIS measurements, whose errors for faint objects can get as high as $0.2 \mathrm{mag}$ for $I \simeq$ 18 mag (Epchtein et al. 1997). A larger difference is found when comparing our $R I$ photometry with the results of CRN99 and CNK00: our results yield systematically fainter magnitudes by about 0.4 mag in average in both passbands. This is not surprising, given that the photometry of CRN99 and CNK00 was calibrated with few secondary standards in the observed field, which are probably variable, and without a colour correction. Because our error seems to be approximately equal for both the $R$ and $I$ photometry, our $R-I$ colours should be very little affected by the exposure time effect.

Because no standard stars are available for the mediumband filters, no absolute calibration was possible for the $\mathrm{H} \alpha$, M 855 and M 915 photometry, and only instrumental magnitudes will be considered for the data in these filters. We applied a standard correction for atmospheric extinction, using the extinction coefficients of the $R$ and $I$ band for the $\mathrm{H} \alpha$ and the two $M$ filters, respectively. For a better understanding of the emission properties, the $\mathrm{H} \alpha$ magnitudes were then shifted so that the main locus of the objects in each surveyed field corresponds to $\mathrm{H} \alpha-R=0$ in a $(\mathrm{H} \alpha, \mathrm{H} \alpha-R)$ colour-magnitude diagram (see Sect. 3 below).

To estimate the completeness limits of our survey, we constructed the number distribution of the found objects as a function of their measured magnitudes for our four WFI fields. These distributions peak for values of $R \simeq 20 \mathrm{mag}$ and $I \simeq$ $19 \mathrm{mag}$, except for the field ChaI-4, where these values are shifted $\lesssim 1$ mag towards fainter objects for both filters. There is a sharp drop in the number of objects for $R \simeq 23 \mathrm{mag}$ ( $22 \mathrm{mag}$ for ChaI-7, due to the shorter exposure times) and $I \simeq 21 \mathrm{mag}$, respectively. Inspection of the corresponding images shows that objects fainter than these values are most likely noise fluctuations. Hence, we consider general detection limits of about 23 mag and 21 mag for the $R$ and $I$ filters, respectively, and completeness limits of $R \simeq 20 \mathrm{mag}$ and $I \simeq 19 \mathrm{mag}$, although the survey might be complete for fainter objects in the area covered by the field ChaI- 4 .

\section{Survey results}

\subsection{Brown dwarf candidates}

At an early stage of their evolution, brown dwarf candidates in star forming regions are easily identified in $(I, R-I)$ colour-magnitude diagrams. Figure 2 shows our diagram for Chamaeleon I. In the already surveyed region around the 
Table 3. Photometry and spectral types of the well known objects in Chamaeleon I ${ }^{a b}$.

\begin{tabular}{|c|c|c|c|c|c|c|c|c|c|c|}
\hline Name & $\alpha(2000)$ & $\delta(2000)$ & $R$ & $I$ & $\mathrm{H} \alpha$ & M 855 & M915 & $S p T^{c}$ & $S p T^{d}$ & Classification \\
\hline CHXR 78C & 110854.7 & -773212.3 & 17.50 & 15.12 & 17.23 & 18.92 & 18.22 & M 5.5 & M 5.5 & star \\
\hline CHXR 21 & 110711.8 & -774637.8 & 15.28 & 13.46 & 15.04 & 17.24 & 16.86 & K 7 & $<\mathrm{M} 4$ & star \\
\hline CHXR 22 & 110713.7 & -774350.3 & 17.21 & 14.96 & 16.94 & 18.65 & 18.14 & $\mathrm{~K} 7+\mathrm{M} 2$ & M4 & star \\
\hline CHXR 74 & 110657.8 & -774211.2 & 15.92 & 13.91 & 15.59 & 17.70 & 17.20 & M 4.5 & M4 & star \\
\hline HM 19 & 110816.9 & -774437.8 & 15.12 & 13.31 & 14.88 & 17.18 & 16.76 & M 3.5 & $<\mathrm{M} 4$ & star \\
\hline Sz 23 & 110758.5 & -774242.0 & 16.55 & 14.64 & 15.59 & 18.43 & 18.03 & M 2.5 & $<\mathrm{M} 4$ & star \\
\hline $\mathrm{ChaH} \alpha 1$ & 110717.2 & -773553.5 & 19.45 & 16.62 & 18.95 & 20.54 & 19.40 & M 7.5 & M9 & $\mathrm{BD}$ \\
\hline $\mathrm{ChaH} \alpha 2$ & 110742.9 & -773400.2 & 17.96 & 15.42 & 17.15 & 19.19 & 18.44 & M 6.5 & M6 & trans. obj. \\
\hline $\mathrm{ChaH} \alpha 3$ & 110752.7 & -773657.6 & 17.80 & 15.26 & 17.61 & 19.15 & 18.30 & M7 & M 6.5 & trans. obj. \\
\hline $\mathrm{ChaH} \alpha 4$ & 110819.4 & -773917.8 & 17.08 & 14.74 & 16.85 & 18.63 & 17.89 & M6 & M6 & trans. obj. \\
\hline $\mathrm{ChaH} \alpha 5$ & 110824.6 & -774148.1 & 17.56 & 15.02 & 17.36 & 18.89 & 18.06 & M6 & M 6.5 & trans. obj. \\
\hline $\mathrm{ChaH} \alpha 8$ & 110746.5 & -774009.6 & 18.37 & 15.78 & 18.22 & 19.67 & 18.79 & M 6.5 & M7 & $\mathrm{BD}$ \\
\hline $\mathrm{ChaH} \alpha 9$ & 110719.2 & -773252.4 & 20.6; & 17.65 & 19.95 & 21.30 & 20.46 & M6 & M 6.5 & trans. obj. \\
\hline $\mathrm{ChaH} \alpha 10$ & 110824.5 & -773930.7 & 19.86 & 17.25 & 19.74 & 21.09 & 20.22 & M 7.5 & M7 & $\mathrm{BD}$ \\
\hline $\mathrm{ChaH} \alpha 11$ & 110829.7 & -773920.4 & 20.4; & 17.72 & 19.89 & 21.59 & 20.65 & M 8 & M7 & $\mathrm{BD}$ \\
\hline $\mathrm{ChaH} \alpha 12$ & 110638.4 & -774309.7 & 18.78 & 16.09 & 18.45 & 19.96 & 18.96 & M7 & M 8 & $\mathrm{BD}$ \\
\hline $\mathrm{ChaH} \alpha 13$ & 110817.5 & -774412.5 & 16.95 & 14.52 & 16.64 & 18.41 & 17.59 & M5 & M 6.5 & trans. obj. \\
\hline
\end{tabular}

a $\mathrm{H} \alpha, \mathrm{M} 855$ and M915 magnitudes are instrumental magnitudes (see text).

$b$ Photometric errors: blank: 0.05; semicolon: $0.1 \mathrm{mag}$; colon: 0.2 mag.

$c$ Spectral types from CNK00 (brown dwarfs and brown dwarf candidates) and CRN99 (stars).

${ }^{d}$ Spectral types from this work. The estimated errors are 1 subclass in the range M 6-M 9 and 2 subclasses in the range M 4-M 6 (see text).

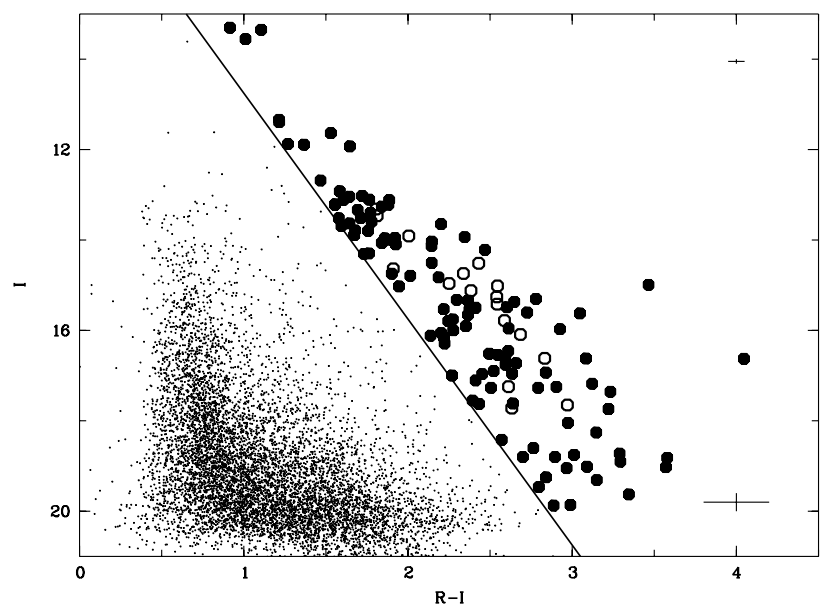

Fig. 2. $(I, R-I)$ colour-magnitude diagram for our surveyed field in Chamaeleon I. The previously identified low-mass stars and brown dwarfs from CRN99 and CNK00 (open symbols) define an empirical isochrone towards redder colours, where we select our new candidates (solid symbols). The crossed lines indicate the average errors.

star HD 97048, we recover all the previously known objects from CRN99 and CNK00 (except for two of them that happened to fall in the interchip gaps): they lie in the region $I \lessgtr 14$ and are clearly separated from background objects towards red$\operatorname{der}(R-I)$ colours. Our optical photometry for these objects is summarized in Table 3 . These previously known objects have an estimated age of about 1-3 Myr, and define an empirical isochrone in the colour-magnitude diagram, which can be used to select candidates in a much larger field. Such an isochrone contains information about the distance and reddening of the objects in the dark cloud. Moreover, by using an empirical rather than a theoretical isochrone, we avoid the uncertainties still present in the evolutionary models for very young ages (see Baraffe et al. 2002 for a critical discussion).

Using such an isochrone, we find 113 candidates members of Chamaeleon I in the same region of the diagram and its prolongation towards fainter sources. The solid line in Fig. 2 is a fit on the brown dwarfs and brown dwarf candidates from CNK00, shifted to allow for the spread observed in the previously known objects and for the estimated photometric errors. Our selected candidates cover the magnitude range down to $I \simeq 20 \mathrm{mag}$. The objects tend to be clustered around the cloud cores (see Fig. 1). The region most devoid of candidate objects lies towards the south of the surveyed area, where only one object is found in the zone of interest of the diagram.

Given the proximity of Chamaeleon I ( $160 \mathrm{pc})$, we do not expect a significant contamination from foreground objects among our candidates. Moreover, due to its high galactic latitude $(b \sim-16)$ and to extinction through the dark cloud, we should not be able either to see many background objects. To estimate the amount of contaminating objects in our field, we made use of the simulations of stellar population synthesis in the Galaxy by Robin \& Crezé (1986) ${ }^{4}$. Within a solid angle of 1 square degree in the direction of the Chamaeleon

\footnotetext{
${ }^{4}$ The simulations can be generated on-line at this URL: http://www.obs-besancon. fr/www/modele/modele_ang.html
} 


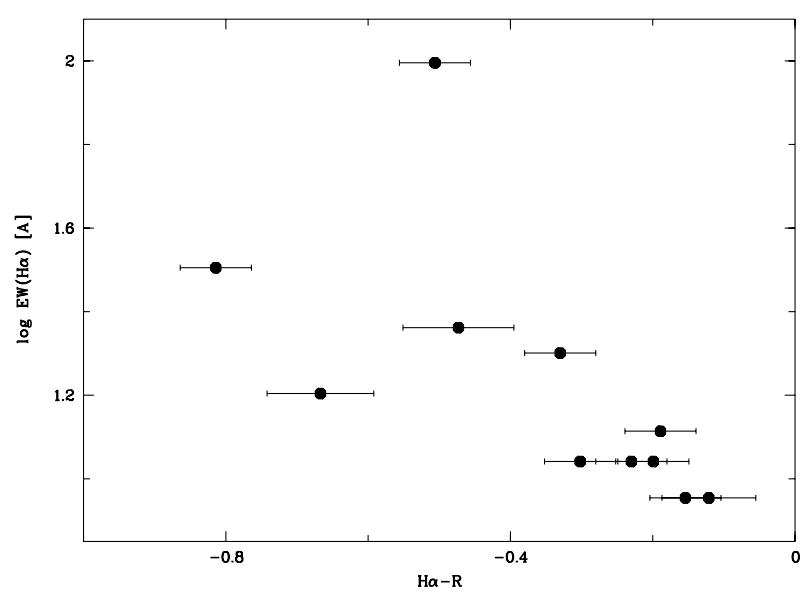

Fig. 3. Logarithm of the $\mathrm{H} \alpha$ equivalent width versus the $(\mathrm{H} \alpha-R)$ colour index for the objects in Chamaeleon I with measured equivalent width. The data are taken from CRN99 for the low-mass stars, and from CNK00 for the brown dwarfs and brown dwarf candidates.

Complex, these simulations yield a number of 5 to 7 objects in the region of the $(I, R-I)$ colour-magnitude diagram around a $1 \mathrm{Myr}$ isochrone from the evolutionary models of Chabrier et al. (2000). Since we find more than hundred objects in the same region of the diagram, we are confident that about $95 \%$ of our candidates are true members of Chamaeleon I.

We note that our survey also includes a number of detections in the $I$-band that have no counterpart in $R$. Most of them lie near the lower magnitude limit of our survey with values of $I=20-21$. These objects may be very red, but because of their non-detection in $R$ nothing can be said about their nature.

According to the theoretical models (Baraffe et al. 1998; Chabrier et al. 2000), if our candidates are indeed members of the star forming region, they should reach down to very low masses, extending down to the deuterium burning limit for the faintest objects in our sample. One way to confirm cloud membership is the detection of $\mathrm{H} \alpha$ emission, which is usually considered as an indicator of youth. All the objects from CRN99 and CNK00 indeed show the $\mathrm{H} \alpha$ line in emission in their spectra.

The amount of emission is usually quantified by the measurement of the $\mathrm{H} \alpha$ equivalent width $E W(\mathrm{H} \alpha)$. Figure 3 shows a plot of $\log E W(\mathrm{H} \alpha)$ versus our measured $(\mathrm{H} \alpha-R)$ colour index for the previously known brown dwarf and brown dwarf candidates. The values of $E W(\mathrm{H} \alpha)$ are taken from CNK00. With the notable exception of $\mathrm{ChaH} \alpha 1$, that will be later discussed in Sect. 6.1, a tendency of smaller equivalent width towards higher colour index can be clearly seen. Of course, one has to be careful with such estimations, because the equivalent width measurements of CNK00 were performed at an epoch different from our optical observations, and these objects are known to have variable $\mathrm{H} \alpha$ emission. However, still the $(\mathrm{H} \alpha-R)$ colour can be used as a rough estimate of the $\mathrm{H} \alpha$ emission.

In Fig. 4, we present a $(\mathrm{H} \alpha, \mathrm{H} \alpha-R)$ colour-magnitude diagram of the objects in our field. The objects from CRN99 and $\mathrm{CNK} 00$ are found in the left part of the diagram, having

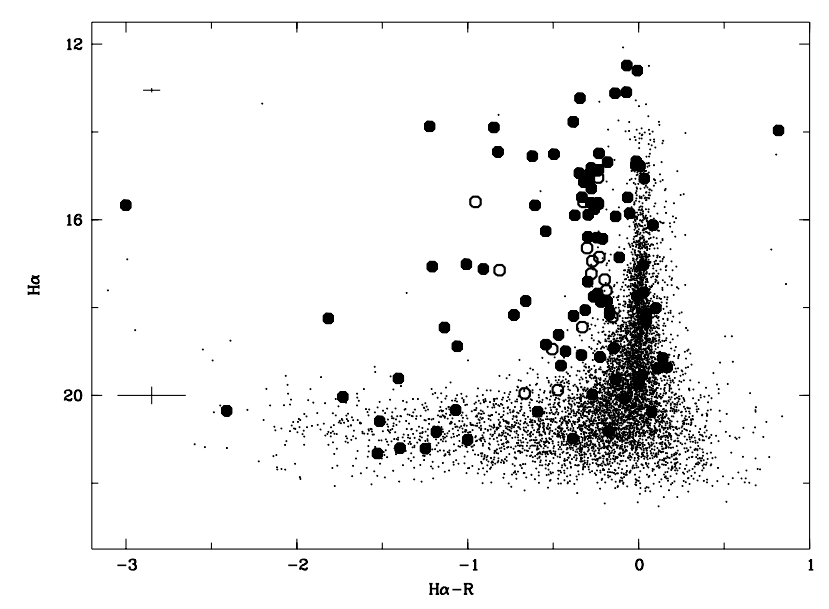

Fig. 4. $(\mathrm{H} \alpha, \mathrm{H} \alpha-R)$ colour-magnitude diagram for our surveyed field in Chamaeleon I. Symbols as in Fig. 2. Objects with $\mathrm{H} \alpha$ emission are placed in the left part of the diagram.

$\mathrm{H} \alpha-R$ colours less than -0.1 . Most of our bright candidates are also located in this region of the diagram, and thus have $\mathrm{H} \alpha$ in emission. Unfortunately, this cannot be clearly stated for most of the faintest objects, due to the large photometric errors in the lower part of our diagram. However, some of them have such extreme measured $(\mathrm{H} \alpha-R)$ colours that they are likely $\mathrm{H} \alpha$ emitters. Moreover, many of them also show an infrared excess (see Sect. 3.3), another indicator that they are probably young. Thus, all the candidates with $\mathrm{H} \alpha-R<-0.1$ in Fig. 4 are considered probable Chamaeleon I members. In this way, we retain 69 objects. Of course, some of the candidates without apparent $\mathrm{H} \alpha$ emission will also belong to the cloud, but this cannot be proved without spectra.

We later added three other objects to this list: ChaI 608, in spite of being slightly too blue for our initial selection, is the probable optical counterpart of the X-ray source CHXR 30 (Feigelson et al. 1993). ChaI 404 has no clear $\mathrm{H} \alpha$ emission, but it could be the optical counterpart of an ISOCAM source (see Sect. 3.3). ChaI 616 has a reported infrared excess (see Sect. 3.3). The coordinates and cross-identifications of all 72 objects are listed in Table 4.

\subsection{Spectral types}

\subsubsection{A photometric classification}

To confirm the membership of our candidates to the Chamaeleon I cloud, we should determine their spectral type. Since there are many objects spread over a large region, and therefore spectroscopy is not easy, we attempt to identify latetype objects exclusively from photometric observations.

M-dwarfs are usually classified by means of their $\mathrm{TiO}$ and VO absorption features, that get deeper with later M-subspectral type (Kirkpatrick et al. 1991; Martín et al. 1999), but disappear in K- and L-type objects. While TiO bands begin to saturate for objects of spectral type later than M 5, VO features become very prominent for late M-type objects. The WFI has a medium-band filter, M 855, covering some important $\mathrm{TiO}$ and $\mathrm{VO}$ absorption features that get deeper with 
Table 4. Candidate low-mass members of Chamaeleon $\mathrm{I}^{a b}$.

\begin{tabular}{|c|c|c|c|c|c|c|c|c|c|c|}
\hline Name & $\alpha(2000)$ & $\delta(2000)$ & $R$ & $I$ & $\mathrm{H} \alpha$ & M 855 & M915 & $S p T^{c}$ & Classification & $\overline{\text { Other Names }^{d}}$ \\
\hline ChaI 401 & 111159.3 & -773038.2 & 22.9: & 19.9 & 21.3: & 23.2: & 22.9 & L 1.5 & BD cand. & \\
\hline ChaI 403 & 111054.1 & -772501.2 & 21.0; & 18.05 & 19.6; & 21.66 & 20.83 & M 6.5 & trans. obj. & DENIS-P J1110.9-7725 \\
\hline ChaI $404^{e}$ & 111037.0 & -772213.8 & 19.70 & 16.62 & 19.7; & 20.23 & 19.36 & M 6.5 & BD cand. & ISO $250^{f}$ \\
\hline ChaI 405 & 111035.4 & -772205.9 & 18.33 & 15.60 & 18.16 & 19.22 & 18.53 & M 5.5 & star & ISO $250^{f}$ \\
\hline ChaI 406 & 111042.0 & -772048.8 & 20.06 & 17.27 & 18.25 & 21.06 & 20.06 & M 8 (M2) & star & ISO 252 \\
\hline ChaI 408 & 111051.4 & -771804.0 & 16.65 & 14.50 & 16.41 & 18.19 & 17.70 & M4 & star & \\
\hline ChaI 410 & 110819.0 & -773041.4 & 19.42 & 16.90 & 18.99 & 20.74 & 19.85 & M7 (M 5.5) & BD cand. & ISO 138 \\
\hline ChaI 411 & 110822.9 & -773028.4 & 18.02 & 15.65 & 17.01 & 19.42 & 18.75 & M5 & star & KG 45, ISO 143 \\
\hline ChaI 412 & 110953.9 & -772837.2 & 21.4: & 18.26 & 20.33 & 21.98 & 20.87 & M9 (M 4.5) & star & GK 30 , ISO 220 \\
\hline ChaI 417 & 110948.0 & -772629.8 & 18.27 & 16.00 & 17.07 & 19.61 & 19.12 & M4 & star & $\begin{array}{l}\text { Baud 43, KG 101, } \\
\text { ISO } 207\end{array}$ \\
\hline ChaI 418 & 110943.1 & -772558.4 & 18.90 & 15.97 & 18.17 & 19.61 & 18.78 & M 6.5 & trans. obj. & C7-1, KG 95, ISO 200 \\
\hline ChaI 419 & 111002.5 & -772545.9 & 21.0; & 17.74 & 20.4: & 21.32 & 20.44 & M7 & $\mathrm{BD}$ & \\
\hline ChaI 420 & 110813.0 & -77 1913.4 & 22.1: & 19.03 & 20.7: & 22.5 & 21.72 & M 9-9.5 (M 1) & star & $\begin{array}{l}\text { KG 37, ISO 131, } \\
\text { DENIS-P J1108.2-7719 }\end{array}$ \\
\hline ChaI 422 & 110342.5 & -772652.8 & 18.57 & 15.96 & 18.19 & 19.75 & 18.87 & M7 & $\mathrm{BD}$ & ISO 28 \\
\hline ChaI 423 & 110242.5 & -772425.2 & 17.71 & 15.34 & 17.41 & 19.20 & 18.47 & M 6 & trans. obj. & \\
\hline ChaI 424 & 110254.5 & -772257.0 & 22.5: & 19.3 & 21.2: & 23.3: & 22.1 & M 8.5 & $\mathrm{BD}$ & \\
\hline ChaI 425 & 110348.3 & -771957.4 & 16.67 & 14.22 & 16.39 & 18.12 & 17.20 & M 7.5 & $\mathrm{BD}$ & Hn2, ISO 32, DENIS 19 \\
\hline ChaI 427 & 110311.7 & -773636.7 & 22.0: & 18.72 & 21.0: & 22.4 & 21.48 & M9 & $\mathrm{BD}$ & DENIS-P J1103.2-7736 \\
\hline ChaI 428 & 110247.4 & -773808.5 & 22.6: & 19.0 & 21.2: & 22.4 & 21.74 & L0 (M 1) & & DENIS-P J1102.8-7738 \\
\hline ChaI 429 & 110724.8 & -774349.4 & 19.77 & 16.93 & 19.64 & 20.67 & 19.77 & M7 & $\mathrm{BD}$ & KG 10 \\
\hline ChaI 432 & 110615.9 & -773750.5 & 19.07 & 16.46 & 18.92 & 20.02 & 19.50 & M4 & star & $\begin{array}{l}\text { ISO 76, } \\
\text { DENIS-P J1106.3-7737 }\end{array}$ \\
\hline ChaI 433 & 110629.3 & -773733.6 & 18.26 & 15.91 & 18.09 & 19.56 & 19.05 & M4 & star & CHXR73, ISO 78 \\
\hline ChaI 434 & 110945.6 & -774034.0 & 18.02 & 15.37 & 17.75 & 19.22 & 18.37 & M7 & $\mathrm{BD}$ & KG 96, ISO 201 \\
\hline ChaI 436 & 111122.8 & -774543.6 & 21.8: & 18.75 & 20.0 & 22.6 & 21.50 & M 8.5 & $\mathrm{BD}$ & \\
\hline ChaI 438 & 111204.0 & -772601.5 & 18.51 & 16.29 & 17.85 & 20.08 & 19.46 & M5 & star & ISO 282 \\
\hline ChaI 441 & 110901.7 & -772053.1 & 21.0: & 18.42 & 20.8: & 22.1 & 21.63 & $\mathrm{~L} 1$ & $\mathrm{BD}$ & ISO 171 \\
\hline ChaI 448 & 110721.0 & -772941.0 & 15.10 & 13.22 & 14.86 & 17.11 & 16.59 & M4 & star & ISO 99 \\
\hline ChaI 449 & 110543.6 & -772652.3 & 15.85 & 13.65 & 15.61 & 17.54 & 16.84 & M 6 & trans. obj. & CHXR15, ISO 65 \\
\hline ChaI 450 & 110644.1 & -772635.0 & 14.87 & 13.11 & 14.69 & 16.94 & 16.56 & $<\mathrm{M} 4$ & star & $\begin{array}{l}\text { T22, UX Cha, ISO } 80 \text {, } \\
\text { DENIS } 38\end{array}$ \\
\hline ChaI 452 & 110659.7 & -771854.3 & 14.74 & 13.02 & 13.90 & 16.72 & 16.49 & $<\mathrm{M} 4$ (M 1.5) & star & T23, UY Cha \\
\hline ChaI 453 & 110233.3 & -772913.6 & 14.72 & 13.11 & 14.49 & 17.03 & 16.69 & $<\mathrm{M} 4$ (M3) & star & $\begin{array}{l}\text { CHXR71, Hn1, } \\
\text { ISO 4, DENIS } 16\end{array}$ \\
\hline ChaI 456 & 110255.7 & -772151.5 & 15.17 & 13.40 & 14.56 & 17.35 & 16.77 & M 5 (M 0.5$)$ & star & T12, Sz10, DENIS 17 \\
\hline ChaI 457 & 110443.1 & -774157.7 & 15.82 & 13.96 & 15.49 & 17.82 & 17.36 & M4 & star & B18, DENIS 26 \\
\hline ChaI 601 & 111231.6 & -765335.2 & 19.35 & 16.76 & 19.12 & 20.75 & 19.72 & M 8 & $\mathrm{BD}$ & \\
\hline ChaI 602 & 110929.7 & -765918.6 & 18.04 & 15.79 & 17.85 & 19.67 & 19.01 & M5 & star & \\
\hline ChaI 603 & 110457.6 & -771557.2 & 16.65 & 14.74 & 16.43 & 18.51 & 18.05 & M4 & star & $\begin{array}{l}\text { T16, Sz13, ISO 55, } \\
\text { DENIS } 29\end{array}$ \\
\hline ChaI 607 & 110812.1 & -771854.0 & 22.8: & 19.9 & 20.4 & 23.2: & 22.6 & L0 (M 0.5) & star & $\begin{array}{l}\text { KG36, ISO 130, } \\
\text { DENIS-P J1108.2-7718 }\end{array}$ \\
\hline Chal $608^{g}$ & 110800.6 & -771731.2 & 17.79 & 15.51 & 17.82 & 19.26 & 18.72 & M4 & star & $\begin{array}{l}\text { CHXR 30, KG 30, } \\
\text { DENIS } 58\end{array}$ \\
\hline
\end{tabular}

later spectral type in M-objects (see Fig. 5 in Kirkpatrick et al. 1991). Another medium-band filter, M915, lies in a wavelength range which is relatively featureless in late-type objects. For the calibration, several objects with known spectral type from K 7 to L 3 were observed, and a correlation was established between the spectral type and the (M 855-M 915) colour index.
We selected our spectral calibrators mostly from the Gliese-Jahreiss catalogue (Jahreiss, private communication). Suitable objects must be observable from the southern hemisphere, not form multiple systems, and not be variable. Unfortunately, the catalogue does not contain many stars of the latest spectral types (M 8-L 3), and very few fulfilled the required conditions. For this reason, we also selected some 
Table 4. continued.

\begin{tabular}{|c|c|c|c|c|c|c|c|c|c|c|}
\hline Name & $\alpha(2000)$ & $\delta(2000)$ & $R$ & $I$ & $\mathrm{H} \alpha$ & M 855 & M915 & $S p T^{c}$ & Classification & Other Names $^{d}$ \\
\hline ChaI 609 & 110757.8 & -771726.9 & 19.78 & 17.27 & 19.3; & 20.91 & 20.32 & M5 & star & $\begin{array}{l}\text { Baud 38, KG 26, } \\
\text { DENIS 55 }\end{array}$ \\
\hline ChaI 610 & 110800.5 & -771532.4 & 18.09 & 15.49 & 17.89 & 19.34 & 18.47 & M7 & BD & KG 29, ISO 121 \\
\hline ChaI 613 & 110827.1 & -771555.8 & 19.38 & 16.73 & 18.84 & 20.60 & 19.62 & M 8 & $\mathrm{BD}$ & ISO 147 \\
\hline ChaI 615 & 110905.7 & -770959.0 & 16.02 & 14.09 & 15.76 & 17.99 & 17.45 & M 4 & star & $\begin{array}{l}\text { Hn7, ISO } 174, \\
\text { DENIS } 71\end{array}$ \\
\hline Chal $616^{e}$ & 110958.8 & -765915.5 & 14.78 & 13.22 & 14.76 & 17.11 & 16.78 & $<\mathrm{M} 4$ & star & KG 113, ISO 229 \\
\hline ChaI 618 & 110650.4 & -765929.1 & 16.97 & 15.03 & 16.86 & 20.15 & 20.00 & $<\mathrm{M} 4$ & star & \\
\hline ChaI 619 & 110437.2 & -770214.5 & 13.26 & 11.89 & 13.12 & 15.77 & 15.53 & $<\mathrm{M} 4$ & star & \\
\hline ChaI 622 & 110515.2 & -77 1129.7 & 15.10 & 13.26 & 14.82 & 17.09 & 16.65 & M4 & star & Hn4, DENIS 30 \\
\hline ChaI 625 & 111029.1 & -771700.4 & 16.18 & 14.04 & 15.89 & 17.92 & 17.21 & M 6 & trans. obj. & Hn12, DENIS 94 \\
\hline Chal 629 & 111123.3 & -770554.7 & 15.87 & 13.95 & 15.64 & 17.80 & 17.36 & $<\mathrm{M} 4$ & star & \\
\hline ChaI 708 & 110913.9 & -762840.1 & 15.89 & 13.99 & 15.61 & 18.03 & 17.43 & M 5 & star & $\begin{array}{l}\text { Hn } 8, \text { KG } 75, \\
\text { DENIS } 73\end{array}$ \\
\hline ChaI 709 & 110851.0 & -762513.8 & 16.81 & 14.79 & 16.26 & 18.87 & 18.20 & M 5.5 & star & $\begin{array}{l}\text { T37, Sz28, KG 54, } \\
\text { ISO 157, DENIS } 68\end{array}$ \\
\hline ChaI 710 & 111022.3 & -762514.2 & 19.09 & 16.54 & 18.62 & 20.66 & 19.50 & M9 & BD & \\
\hline ChaI 711 & 110711.9 & -762550.2 & 18.37 & 16.15 & 18.06 & 20.01 & 19.32 & M 5.5 & star & \\
\hline ChaI 712 & 110632.8 & -762521.0 & 19.42 & 16.96 & 19.1; & 20.93 & 19.92 & M 8 & $\mathrm{BD}$ & \\
\hline ChaI 717 & 110802.5 & -764034.3 & 17.92 & 15.54 & 17.68 & 19.58 & 18.68 & M7 & $\mathrm{BD}$ & \\
\hline ChaI 721 & 110951.6 & -764545.4 & 16.06 & 14.30 & 15.92 & 18.09 & 17.78 & $<\mathrm{M} 4$ & star & ISO 214 \\
\hline ChaI 726 & 110952.2 & -763913.0 & 19.59 & 16.96 & 18.45 & 20.87 & 19.92 & M7.5 & $\mathrm{BD}$ & $\begin{array}{l}\text { GK } 29, \text { KG } 106, \\
\text { ISO } 217\end{array}$ \\
\hline ChaI 731 & 110922.8 & -763432.2 & 19.94 & 17.55 & 18.88 & 21.11 & 20.74 & $<\mathrm{M} 4$ & star & C1-6, NIR 10, ISO 189 \\
\hline ChaI 735 & 110855.1 & -763240.1 & 18.03 & 15.76 & 17.12 & 19.63 & 18.95 & M 5.5 & star & $\begin{array}{l}\text { C1-25, NIR 19, KG 59, } \\
\text { ISO } 165\end{array}$ \\
\hline ChaI 737 & 110851.9 & -763250.3 & 20.3; & 17.61 & 20.0; & 21.60 & 20.62 & M8 & BD & \\
\hline ChaI 742 & 111203.4 & -763703.7 & 16.28 & 14.13 & 15.90 & 18.18 & 17.43 & M6 & trans. obj. & $\begin{array}{l}\text { Hn16, CHXR 84, } \\
\text { DENIS } 105\end{array}$ \\
\hline ChaI 743 & 111145.4 & -763650.1 & 21.4: & 18.60 & 21.0: & 22.8 & 21.47 & M 8 & $\mathrm{BD}$ & \\
\hline ChaI 747 & 111324.6 & -762923.2 & 15.29 & 13.69 & 14.94 & 17.67 & 17.32 & $<\mathrm{M} 4$ & star & Hn18, DENIS 116 \\
\hline ChaI 748 & 111329.8 & -762901.7 & 15.23 & 13.51 & 14.94 & 17.48 & 17.06 & $<\mathrm{M} 4$ & star & Hn19, DENIS 118 \\
\hline ChaI 749 & 111259.4 & -761653.6 & 13.57 & 11.93 & 13.23 & 15.98 & 15.63 & $<\mathrm{M} 4$ & star & \\
\hline ChaI 751 & 110642.0 & -763549.0 & 15.28 & 13.63 & 14.45 & 17.61 & 17.13 & M4 & star & \\
\hline ChaI 752 & 110840.8 & -763607.8 & 14.15 & 12.68 & 13.77 & 16.65 & 16.38 & $<\mathrm{M} 4$ (M 2) & star & $\begin{array}{l}\text { CHX 13a, KG 52, } \\
\text { ISO 153, DENIS } 65\end{array}$ \\
\hline ChaI 753 & 110953.5 & -763425.5 & 15.10 & 13.52 & 13.87 & 17.31 & 17.09 & $<\mathrm{M} 4$ & star & \\
\hline ChaI 754 & 111248.8 & -764706.7 & 15.46 & 13.79 & 15.15 & 17.78 & 17.34 & $<\mathrm{M} 4$ & star & \\
\hline ChaI 755 & 111056.2 & -764532.7 & 16.28 & 13.93 & 15.67 & 17.94 & 17.02 & M7 & $\mathrm{BD}$ & \\
\hline ChaI 757 & 111210.0 & -763436.8 & 15.00 & 13.12 & 14.51 & 17.18 & 16.60 & M4 & star & \\
\hline ChaI 759 & 111333.7 & -763537.8 & 15.37 & 13.59 & 15.08 & 17.57 & 17.11 & M 4 & star & \\
\hline ChaI 760 & 111011.5 & -763529.3 & 15.56 & 13.89 & 15.29 & 17.74 & 17.47 & $<\mathrm{M} 4$ (M 1.5) & star & $\begin{array}{l}\text { NIR 45, KG 121, } \\
\text { ISO 237, } \\
\text { DENIS-P J1110.2-7635 }\end{array}$ \\
\hline
\end{tabular}

${ }^{a} \mathrm{H} \alpha, \mathrm{M} 855$ and M915 magnitudes are instrumental magnitudes (see text).

$b$ Photometric errors: blank: 0.05; semicolon: $0.1 \mathrm{mag}$; colon: 0.2 mag.

${ }^{c}$ The estimated errors in the spectral types are 1 subclass in the range M 6-M 9 and 2 subclasses in the range M 4-M 6 (see text). Spectral types from the literature are indicated in parenthesis.

$d$ References: Sz\#: Schwartz (1977); Hn\#: Hartigan (1992); T\#: Schwartz (1991); CHXR\#: Feigelson et al. (1993); C\#: Prusti et al. (1991); NIR\#: Oasa et al. (1999); Baud\#: Baud et al. (1984); CHX\#: Feigelson \& Kriss 1989; B\#: Lawson et al. (1996); ISO\#: Persi et al. (2000), P. Persi, priv. communication; DENIS\#: Cambrésy et al. (1998, Table 1); DENIS-P\#: Cambrésy et al. (1998, Table 2); GK\#: Gómez \& Kenyon (2001); KG\#: Kenyon \& Gómez (2001).

$e$ This object does not have $\mathrm{H} \alpha$ emission according to its $(\mathrm{H} \alpha-R)$ colour.

$f$ Ambiguous counterpart (see text).

$g$ The $(R-I)$ colour of this object is slightly too blue according to our selection criteria.

objects from the LHS catalogue (Luyten 1979) and from the surveys DENIS (Delfosse et al. 1999; Martín et al. 1999) and 2MASS (Kirkpatrick et al. 2000), although in these cases we could not be sure that the objects were single-star systems.
A first set of calibrators was observed in two nights (on the 30th and 31st of May 1999) of the observing run in which our Chamaeleon I fields were taken. Once the feasibility of our method was demonstrated, a second and larger set was observed on the 23rd December 2001 to improve our 
Table 5. Our sample of spectral standards ${ }^{a b}$.

\begin{tabular}{lccccc}
\hline \hline Name $^{c}$ & $\alpha(2000)$ & $\delta(2000)$ & M 855 & M 915 & SpT \\
\hline GJ 13460 & 092851 & -091600 & 13.12 & 13.19 & K 7 \\
GJ 7 59 & 065142 & -435312 & 12.96 & 13.02 & M 0 \\
GJ 494 & 130047 & 122233 & 11.83 & 11.69 & M 0.5 \\
GJ 7 58 & 063950 & -612848 & 12.71 & 12.77 & M 1 \\
GJ 22066 & 081608 & 011812 & 12.24 & 12.26 & M 2 \\
GJ 4 659 & 073841 & -211330 & 13.59 & 13.56 & M 3 \\
GJ 402 & 105052 & 064829 & 12.97 & 12.64 & M 3 \\
GJ 4 512 & 053600 & -073900 & 14.40 & 14.20 & M 4 \\
GJ 21055 & 030900 & 100124 & 15.99 & 15.69 & M 5 \\
GJ 4 195 & 020216 & 102018 & 16.19 & 15.73 & M 6 \\
LHS 3003 & 145638 & -280950 & 16.57 & 15.72 & M7 \\
GJ 5 14 & 025226 & 005618 & 19.81 & 19.27 & M 8 \\
LHS 2065 & 085336 & -032932 & 18.48 & 17.40 & M9 \\
GJ 4 619 & 070753 & -490048 & 20.22 & 19.42 & M9 \\
DENIS-P J0909.9-0658 & 090957 & -065806 & 20.67 & 20.19 & L 0 \\
2MASSW J0832045-012835 & 083205 & -012835 & 20.98 & 20.57 & L 1.5 \\
2MASS J1342236+175156 & 134236 & 175156 & 19.81 & 19.80 & L 2 \\
2MASSW J0928397-16031 & 092840 & -160312 & 21.30 & 21.34 & L2 \\
DENIS-P J1058.7-1548 & 105847 & -154800 & 21.18 & 20.71 & L 2.5 \\
DENIS-P J1047.5-1815 & 104731 & -181558 & 21.40 & 20.60 & L 2.5 \\
\hline
\end{tabular}

a M 855 and M 915 magnitudes are instrumental magnitudes.

$b$ Photometric errors: blank: 0.05; semicolon: $0.1 \mathrm{mag}$; colon: 0.2 mag.

c References: GJ\#: H. Jahreiss (private communication); LHS\#: Luyten (1979); DENIS: Delfosse et al. (1999), Martín et al. (1999); 2MASS: Kirkpatrick et al. (2000).

initial calibration. In total, we observed 22 objects in the M 855 and M915 filters, but three of them were rejected because they seemed double, or because of technical problems during the observations. Data reduction and aperture photometry were performed in the way explained in Sect. 2.2. Table 5 summarizes our photometry results and other relevant data of our final calibrator list. We recall that no absolute photometric system is available for the M 855 and M 915 passbands. We could not use these objects to establish an absolute magnitude scale in these filters because for most of them the distance is still unknown.

For mid- to late M-type objects (M4-M9), a correlation between colour and spectral type is clearly found (see Fig. 5): objects with later spectral type also have a higher (M 855M915) colour index. This is not seen for early M-type objects, because the features considered are not prominent in their spectra. The observed scatter probably is due mainly to differences in age (see also Sect. 3.2.2); metallicity differences may also play a role. The best fit obtained in this range is:

$S p T=(6.2 \pm 1.2) \cdot(\mathrm{M} 855-\mathrm{M} 915)+(2.8 \pm 0.7)$

where $S p T$ is an integer (4-9) denoting the M subspectral class. The quoted errors are the standard deviations of the corresponding coefficients.

For early L-type objects (L 0-L 2), an anticorrelation is observed, as expected from the progressive weakening of the $\mathrm{TiO}$

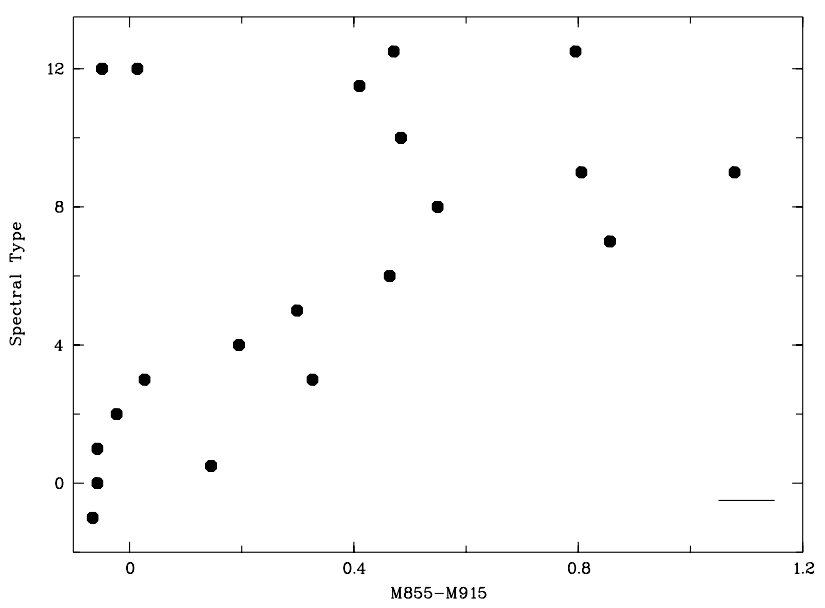

Fig. 5. Spectral type versus (M 855-M 915) colour index for our set of spectral calibrators. Numbers from 0 to 9 correspond to the M subspectral classes, and from 10 on to the $\mathrm{L}$ spectral sequence. For midto late M-type objects (M4-M9), a correlation between colour and spectral type is clearly seen. The bar at the lower right corner of the plot shows the average error in the (M 855-M 915) colours.

and VO features. However, this anticorrelation seems to break down for objects of spectral type later than L 2. This is probably due to the appearance of the alkali absorption features, a 


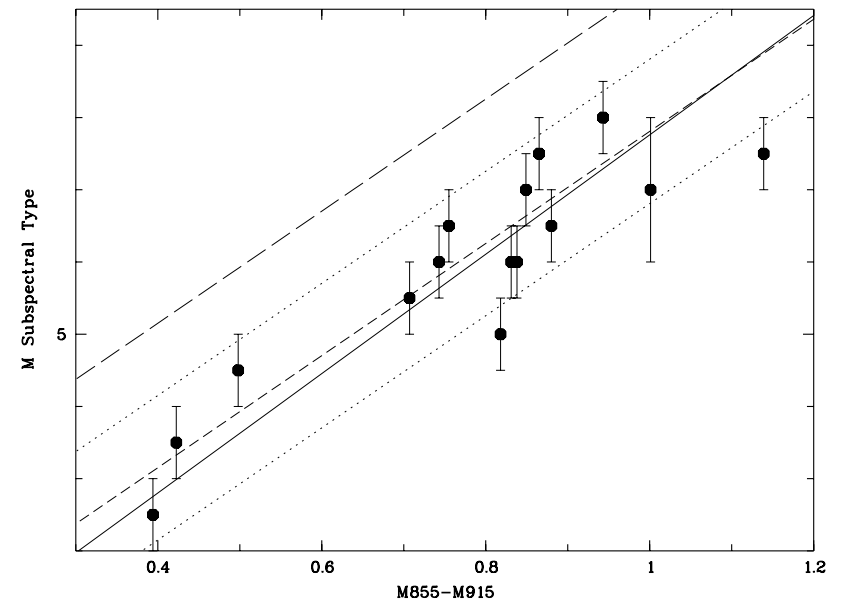

Fig. 6. Spectral type versus (M 855-M 915) colour for the objects in Chamaeleon I with known spectral type. The errors are taken from CNK00. A correlation between colour and spectral type is clearly seen. The long-dashed line is the fit obtained with our spectral calibrators. The solid line is the fit for this second set of objects. Both lines are almost parallel, as indicated by the short-dashed line, which corresponds to the fit on the old objects, but shifted 2 subclasses. The dotted lines indicate the estimated error of about 1 subclass.

characteristic of the $\mathrm{L}$ spectral sequence. One Cs I line lies towards the centre of the wavelength range covered by our M 855 filter, and it starts to be important for L 3 and latertype objects (see Kirkpatrick et al. 1999, their Figs. 4, 6 and 7). Since L 3 is the limit of our observed spectral range, we cannot state whether a sequence similar to that of late M-objects, but based on the alkali absorption features, could be established also for late L-objects. In the range M9-L 2, we obtain as best fit:

$S p T=(-3.1 \pm 0.6) \cdot(\mathrm{M} 855-\mathrm{M} 915)+(12.0 \pm 0.4)$

where $S p T$ is now in the range 9-12 (a value of 10 corresponds to a spectral type $\mathrm{L} 0$ ).

\subsubsection{Application to young objects}

In a next step, we used Eq. (3) to derive spectral types for the known objects in Chamaeleon I.

The results are shown in Fig. 6. The young objects appear to have, according to our fit, later spectral types than the ones derived from spectroscopic observations. However, the trend to increasing colour with later spectral type is clearly reproduced. The solid line shows a fit performed on this set of young objects. This line is almost parallel to the one obtained from the fit to the (old) field objects, just being shifted to earlier spectral types by about 2 subclasses.

We interpret this shift as a consequence of the lower value of $\log g$ for these young objects. Work by several authors (e.g. Martín et al. 1996; Luhman et al. 1997) shows that young M-type objects (characterized by low-gravity atmospheres) display stronger $\mathrm{TiO}$ and $\mathrm{VO}$ absorption than field dwarfs of the same spectral class (high-gravity atmospheres). Stronger TiO and VO absorption implies fainter M 855 magnitudes and redder (M 855-M 915) colours. The age difference should also be responsible, to some extent, for the scatter of our calibrators.
The effect of age (i.e. gravity) in our medium-band photometry can be tested with the help of theoretical models. The left panel of Fig. 7 shows different isochrones in a ( $T_{\text {eff }}$, M 855M915) diagram from the non-grey models of Baraffe et al. (1998; Baraffe, private communication). The represented ages range from 1 to $600 \mathrm{Myr}$. All curves tend to converge for effective temperatures higher than $4000 \mathrm{~K}$, corresponding to late K-type objects. For lower temperatures, the younger objects tend to exhibit redder (M 855-M915) colours than the older ones of same spectral type. The biggest differences are observed in the temperature range between 4000 and $2800 \mathrm{~K}$, roughly corresponding to spectral types from K 7 to M 6. Here, a 1 Myr old object will have the same colour as a $600 \mathrm{Myr}$ old M-dwarf that is about $800 \mathrm{~K}$ cooler. Even compared to a $3 \mathrm{Myr}$ old object a remarkable temperature difference is predicted $(\sim 200 \mathrm{~K})$. However, for cooler objects $\left(T_{\text {eff }} \lesssim 2800 \mathrm{~K}\right)$, the colour difference is no longer significant at very young ages (less than $10 \mathrm{Myr}$ ), and the difference between young and old objects is notably decreased (from 800 to about $150 \mathrm{~K}$ ). On the other hand, the isochrones for intermediate ages (10-70 Myr) appear more or less parallel through the whole represented range. The oldest isochrone ( $600 \mathrm{Myr}$ ) only diverges from them at very low temperatures.

The relation between spectral type and (M 855-M915) colour index can be better understood with the help of the right panel of Fig. 7. Here we have adopted the $T_{\text {eff }}$ scale versus spectral type for pre-main sequence objects from Luhman (1999):

$T_{\text {eff }}=3850-141.0 \cdot S p T$.

It is immediately seen that the behaviour of the curves is not exactly linear: for blue (M 855-M915) colours (earlier spectral types) the difference between old and young objects can be as high as 5 subclasses, but this difference is reduced towards redder colours (later spectral types). If we shift the oldest isochrone 2 subclasses, however, the difference is not greater than 1 subclass for spectral types later than M4. The value of this shift is the approximated offset that we find between our fit to (old) field M-dwarfs and the (young) objects in Chamaeleon I. We therefore conclude that the observed offset between our initial spectral type calibration and the reported spectral types for the objects in Chamaeleon I is mainly due to the difference in age between them and our calibrators, which implies a different value of $\log g$. To account for this effect, we shifted our derived scale 2 subclasses towards earlier spectral types (see Fig. 6). In most of the cases, the so derived spectral types coincide with the ones in CRN99 and CNK00 (obtained from object spectra) with an error of one subclass at most (see Table 3). For early M-type objects, the error is certainly larger, due to the saturation of the (M 855-M 915) colour. We conservatively consider an error of two subclasses in the range $\mathrm{M} 4-\mathrm{M} 6$.

We cannot test our (M 855-M915) colour index for L-dwarfs in the same way, because no L-type objects are yet known in Chamaeleon I. The model curves of Fig. 7 do not go deep enough into the L-type temperature range to test the observed behaviour. Therefore, we can only tentatively assign L 0 to L 2 spectral types to the objects found at the bottom left part of a (M 855, M 855-M915) colour-magnitude diagram. 

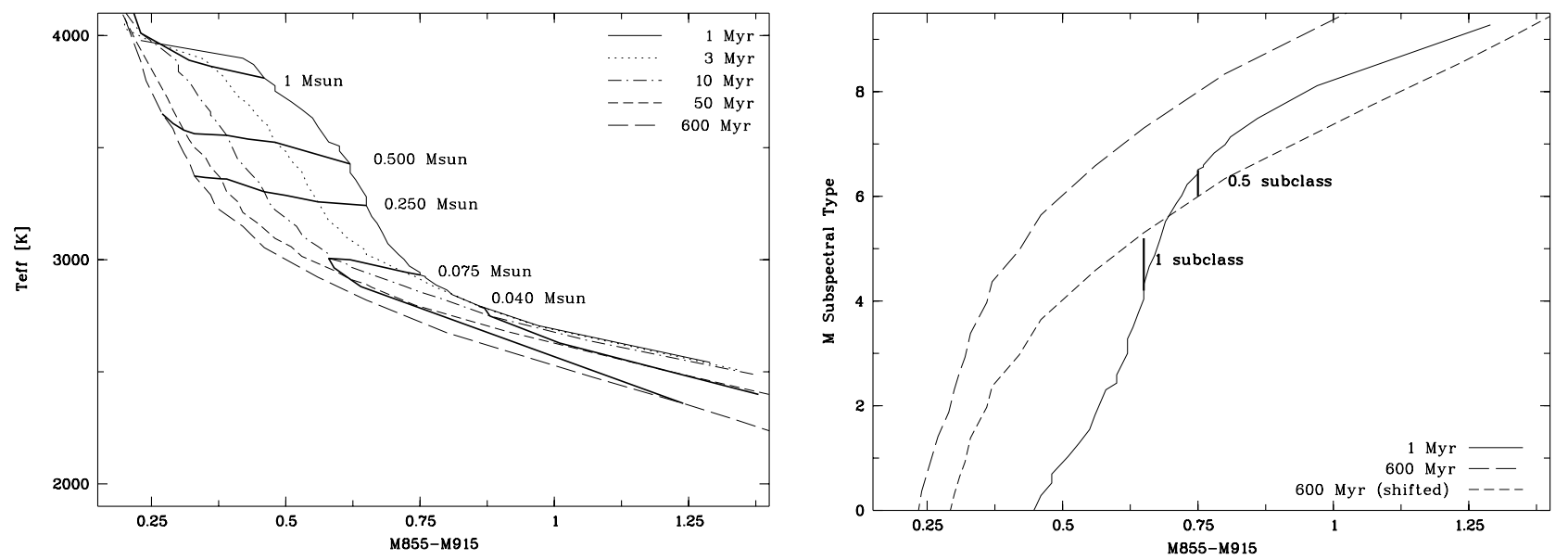

Fig. 7. Left panel: isochrones of effective temperature versus theoretical (M 855-M 915) colour index from the Baraffe et al. (1998) models. The curves of equal mass are also plotted. For the same value of the colour index, older objects have a lower temperature, thus a later spectral type. Hence, by using a fit to old objects, the temperatures and masses of young very low-mass objects are overestimated. Right panel: spectral type versus theoretical (M 855-M 915) colour index for the $1 \mathrm{Myr}$ and the $600 \mathrm{Myr}$ isochrones. At low values of the colour index, older objects are several subclasses later than very young objects. This difference decreases to at most 1 subclass for objects later than M 4 if the oldest isochrone is shifted by 2 subclasses.

We note, however, that highly extincted M-type objects would also fall in the same region of the diagram, making this classification somewhat uncertain. Hence, it is difficult, at the present stage, to distinguish between a L-type object and a rather extincted M-type object with this method (see also the discussion in the following section). A more detailed analysis is required before a reliable determination of the $\mathrm{L}$ subspectral class can be achieved with this photometric technique.

\subsubsection{Spectral types of the brown dwarf candidates}

Figure 8 shows the (M915, M 855-M915) colour-magnitude diagrams for our Chamaeleon I field. Our derived scale for the spectral type identification is also shown. Clearly, many of our candidates have mid to late spectral type M. About one fifth of them have spectral type M7 or later. Given that the stellar/substellar boundary at the age of Chamaeleon I is set at a spectral type of about M 6 by the theoretical models, we classify these objects as brown dwarfs. There is a smaller group of seven or eight objects with spectral types between M 5.5 and $\mathrm{M} 7$, in the transition region from low-mass stars to brown dwarfs. Around 50 objects have spectral types M 5 or earlier, so they are very low-mass stars. For the faintest objects, a turning back towards lower values of the (M 855-M 915) colour is seen in Fig. 8 at spectral type M9, which may be indicative of the beginning of the L-dwarf sequence. If their belonging to the cloud can be confirmed, these objects might be low-mass brown dwarfs.

The photometry results and the derived spectral type for all our candidates are summarized in Table 4 . The values obtained from Eqs. (3) and (4) have been rounded to the closest value in steps of 0.5 subclasses. Although 23 objects from our survey list had already been proposed as cloud members in previous optical and/or X-ray studies of Chamaeleon I (see references in Table 4), many of them still lacked a spectral type determination. We have checked our photometrically derived

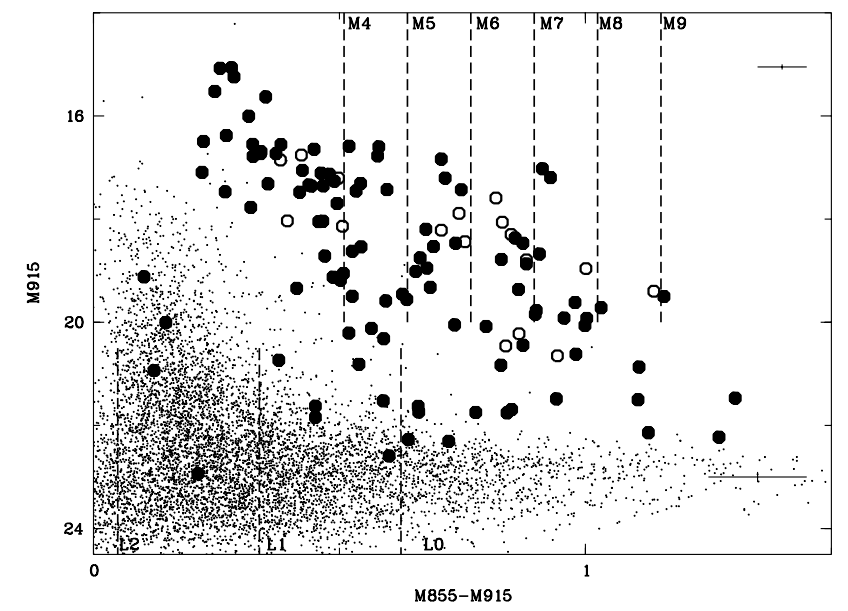

Fig. 8. (M 915, M 855-M 915) colour-magnitude diagram for our surveyed field in Chamaeleon I. Symbols as in Fig. 2. A scale for the identification of the spectral type is also indicated (see text).

spectral types with those given by Lawson et al. (1996) and Gauvin \& Strom (1992) for the objects in common with these authors (7). In general there is good agreement between their and our spectral types, except for the star Sz 10 (ChaI 456), to which Gauvin \& Strom assign a spectral type of M 0.5, while our photometric classification yields a spectral type of M 5 . We do not see, however, any other object near ChaI 456 that could be the one observed by Gauvin \& Strom. DENIS photometry is also available for this object (Cambrésy et al. 1998, see Sect. 3.3 below). The $I$ magnitude measured by these authors is consistent with our own result within the estimated errors, while their $J K$ s photometry agrees well with that given by Gauvin \& Strom (1992). We are thus quite confident that our identification of ChaI 456 with $\mathrm{Sz} 10$ is correct. However, the measured $I$ magnitude (13.40 mag) appears too bright for an M 5 object. The discrepancy could be explained if $\mathrm{Sz} 10$ were an unresolved binary. In that case, Gauvin \& Strom would have 
observed the spectrum of the primary. We would wrongly assign a later spectral type to the unresolved pair, because our spectral type calibration is only based on the (M 855-M 915) colour index (which should be redder for a binary object), and not on magnitudes (which should be brighter). As a matter of fact, in Table 3 we see that CHXR22, a known binary formed by a K 7 and a M 2 object that appear unresolved in our images, has an estimated spectral type of M4 according to our classification. Thus an hypothetical secondary of Sz 10 would probably have a spectral type earlier than M 5, definitively being a star.

More recently, Gómez \& Persi (2002) presented infrared spectroscopic observations of several mid-infrared sources from a previous ISOCAM survey in Chamaeleon I (Persi et al. 2000). Six of their objects are also included in our list of probable cloud members (some of them in common with other authors). They give an error of 2 subclasses for their spectral type classification. While for four of the objects the agreement between their and our spectral types is rather good, for the other two we find again a large discrepancy: ISO 252 (our object ChaI 406) is classified as an M2 object according to these authors, but we derive a spectral type of M 8 for it. Its $I$ magnitude according to our and the DENIS measurement (around $17.2 \mathrm{mag}$ ) is slightly too faint compared to that of other objects of similar spectral type in the cloud. On the other hand, the object ISO 250 (ChaI 405), which lies not too far from ISO 252, has a spectral type of M 6 according to Gómez \& Persi and of M 5.5 according to our own classification, in spite of being brighter than ISO 252 in the $I$ band. The discrepancy between both spectral type classifications for ISO 252 might be due to a locally higher extinction towards this object. A similar situation is seen in the case of ISO 220 (ChaI 412), which we classify as M9 and Gómez \& Persi as M4.5. The I magnitude for this object (greater than 18 mag according to both DENIS and our photometry) is very faint compared to other objects of spectral type M4-M 5 in the cloud (typically between 16 and $17 \mathrm{mag})$. The $(R-I)$ colours of both ChaI 406 and ChaI 412 are too red for early M-type objects, which might indeed hint to the presence of moderate extinction.

We note, however, that the discrepancy between luminosity and spectral type might also be due to the presence of circumstellar dust, as proposed by Fernández \& Comerón (2001) for the object LS-RCrA 1 in Corona Australis. This object appears too faint compared to other objects of similar spectral type and age. More such subluminous objects have been identified in other star forming regions.

The effect of extinction is more clearly seen when comparing our spectral types with those given by Gómez \& Mardones (2003). These authors performed near-infrared spectroscopy of 46 candidate young stellar objects from several photometric surveys in Chamaeleon I. We have six objects in common with this work ${ }^{5}$. For three of them (ChaI 403, ChaI 432 and ChaI 760), the spectral classification given by Gómez $\&$ Mardones agrees with ours within their and our estimated

\footnotetext{
${ }^{5}$ Gómez \& Mardones (2003) actually observed seven of our objects, but the spectrum of one of them, ChaI 427, was too noisy to allow a spectral type classification.
}

errors. This is not the case, however, for the other three objects (ChaI 420, ChaI 428 and ChaI 607), whose spectral types are remarkably warmer according to the spectroscopic data. While we assign them spectral types between M 9 and L 0 with our photometric method, Gómez \& Mardones classify them as early M-type objects (M 0.5-M 1). These authors obtain values between 2 and $5 \mathrm{mag}$ for the extinction $\mathrm{A}_{J}$ in the $J$ band towards these objects, which are among the faintest ones in our survey.

These cases illustrate the limitations of our photometric spectral type determination: we will estimate too late spectral types for some objects that lie in regions of locally high extinction, which could then be wrongly classified as brown dwarfs. The uncertainties are especially large for the faintest candidates in our sample, that could be identified as L-type objects in spite of being highly extincted early M-type stars.

On the other hand, if a particular object is a binary which appears unresolved in our WFI images, we are likely to assign a later spectral type to the combined pair. Nevertheless, among a total of 36 objects with known spectral type, we have only six clear discrepancies, meaning that in about $80 \%$ of the cases our classification is reliable. This reliability is even higher (around 90\%) for the brightest objects $(I<19)$. On these objects, a linear fit of our predicted spectral types versus the ones from the literature gives a rms error of about one subclass, in agreement with our expectations.

Out of 72 objects, we have classified 44 objects as lowmass stars (with spectral type earlier than M6), and 19 objects as brown dwarfs (with spectral type later than M 6.5). We also find 6 transition objects with spectral types between M 6 and M 6.5, which will be considered brown dwarf candidates in the subsequent discussion. Three objects that could be low-mass brown dwarfs of spectral type $\mathrm{L}$ are also detected. However, in some cases the identification of our faint candidates as cloud members is not certain because of the large photometry errors in the lower part of the ( $\mathrm{H} \alpha, \mathrm{H} \alpha-R)$ colourmagnitude diagram and/or the lack of infrared excess (see Sect. 3.3). Moreover, as explained in this section, their spectral type classification may be somewhat uncertain and requires spectroscopic confirmation. We will regard these objects as brown dwarf candidates, although keeping in mind that they might be embedded stars.

In the subsequent analysis we do not include the doubtful objects ChaI 608 and ChaI 404. In the seven cases mentioned above where the photometric spectral type does not match with reported spectroscopic observations, the classification derived from object spectra was preferred. Spectral types from the literature are indicated in parenthesis in the corresponding column of Table 4.

\subsection{Infrared detections}

We cross-correlated our Chamaeleon I object list with published detections by ISOCAM (Persi et al. 2000) and DENIS (Cambrésy et al. 1998), as well as the surveys by Gómez \& Kenyon (2001) and Kenyon \& Gómez (2001). In this way, we got infrared photometry for about $67 \%$ of the objects in our 
sample. Of them, 47 have a near-infrared counterpart and 33 a mid-infrared counterpart.

Persi et al. (2000) provide a list of faint Chamaeleon I members with mid-infrared excesses from ISOCAM observations. We found 19 objects in common in both lists. Another $11 \mathrm{ob}-$ jects from our sample have been detected by ISOCAM in one or both bands (Persi, private communication). The ISOCAM list also provides DENIS IJKs photometry for all the detected sources. In addition, we recover 23 objects with near-infrared excess from DENIS photometry according to Cambrésy et al. (1998). Gómez et al. (2001) report a list of 118 candidate members of the Chamaeleon I cloud with infrared excess from $J H K$ observations, but we find only two objects in common with these authors. Kenyon \& Gómez (2001) provide $J H K s L$ photometry for a sample of young stellar candidates in Cha I, including many from the previous surveys and some new detections. This sample contains 16 of our objects. In total, we find 33 objects with a reported near-infrared excess. For the ones observed by several authors, the photometry results are consistent within the quoted errors. The available infrared photometry for our objects, together with the cross-identifications and references are summarized in Table 6. Surprisingly, only 10 objects exhibit both near- and midinfrared excesses. This represents less than $25 \%$ of the total infrared detections in our sample.

A dubious cross-identification regards the objects ChaI 404 and ChaI 405, which lie relatively close at a distance of about 9.'5. The coordinates given by Persi et al. (2000) for the ISOCAM source ISO 250 match well the position of ChaI 404, the fainter of the two, but the $I$-band photometry from DENIS most probably corresponds to ChaI 405, which is about one magnitude brighter than ChaI 404. In our "reddest" filter, M915, the brightness difference between both sources is reduced to about $0.8 \mathrm{mag}$. It is possible that ChaI 404 is brighter in the infrared than its neighbour, thus leading to an eventual identification error. However, ChaI 404 does not show evident $\mathrm{H} \alpha$ emission according to its $\mathrm{H} \alpha-R$ colour, so its membership to the Chamaeleon I cloud is doubtful according to our selection criteria. Given the ISOCAM pointing errors, we cannot exclude that ChaI 404 is indeed a background object, and that ChaI 405 is the true counterpart to the mid-infrared source.

The detection of infrared excesses indicates that the Chamaeleon I candidate members must be young. However, it should be taken into account that criteria for identifying objects with infrared excess are not homogenous among the different authors. In particular, we note that the Gómez \& Kenyon survey has few objects in common with the other two, in spite of their spatial overlap. For a detailed discussion of the infrared properties, we refer to the quoted works. Nonetheless, something has to be said about the extinction towards our objects, as it may affect their properties, and in particular, according to the previous section, their spectral type classification.

In Fig. 9 we show the near-infrared colour-colour diagram for our candidates with $J H K$ photometry (Gómez \& Kenyon 2001; Kenyon \& Gómez 2001). The solid curve is a 1 Myr isochrone from the Chabrier et al. (2000) models. The dashed and dotted lines indicate the position of this isochrone for extinction values of $A_{V}=5 \mathrm{mag}$ and $A_{V}=10 \mathrm{mag}$, respectively, computed with the law of Rieke \& Lebofsky (1985). The colours of most of our candidates are consistent with extinction values between 0 and $10 \mathrm{mag}$. The three exceptions are ChaI 420, ChaI 607 and ChaI 609, that appear to be significantly reddened. For the two former objects, this result was expected after the discussion from Sect. 3.2.3. Note that for the third object with a remarkably discrepant spectral type (ChaI 428) only DENIS photometry is available. ChaI 609, on the other hand, is very red for its estimated spectral type (M 5).

Persi et al. (2000) used a $(J-K, K-m(6.7))$ colour-colour diagram to separate the effects from intrinsic infrared excess from those of reddening.

The apparent magnitudes $m(6.7)$ and $m(14.3)$ corresponding to the ISOCAM fluxes (in Jy) are computed with the following relations:

$$
\begin{aligned}
& m(6.7)=-2.5 \cdot \log \frac{F_{v}(6.7 \mu \mathrm{m})}{83.4} \\
& m(14.3)=-2.5 \cdot \log \frac{F_{v}(14.3 \mu \mathrm{m})}{18.9}
\end{aligned}
$$

In Fig. 10 we show the position of our objects in the $(J-K$, $K-m(6.7))$ diagram. The solid and dashed lines indicate the location of the reddening band computed by Persi et al. (2000). The objects with intrinsic $(K-m(6.7))$ excess are found to the right of the reddening band. ChaI 420 and ChaI 607 are clearly separated from the rest, having high values of the $(J-K)$ colour index, but low or moderate $(K-m(6.7))$ colours. This diagram confirms that these two objects are heavily reddened, and that many from our candidates suffer from moderate extinction.

\section{Spatial distribution}

Figure 1 shows the positions of our objects the Chamaeleon I region, as well as those of the previously known brown dwarfs and very low-mass stars from CRN99 and CNK00. The distribution of the brown dwarfs and brown dwarf candidates resembles that of the low-mass stars. All these objects are mostly located in the two cloud cores containing the two intermediate mass stars HD 97048 and HD 97300 and at the boundaries of a third core, apparently the densest according to $\mathrm{C}^{18} \mathrm{O}$ observations (Mizuno et al. 1999). This core lies between the other two and is seen as a dark region devoid of stars in the optical images. Far from the three cloud cores, few objects are found.

To better quantify this observed distribution, we have counted the number of objects at different distance bins from the stars HD 97048 and HD 97300. Figure 11 shows the histograms derived from these counts ${ }^{6}$. We do see that the distributions of both stars and brown dwarfs peak near the positions of the intermediate mass stars. In general, the objects seem to

\footnotetext{
6 Although these counts might be somehow incomplete, due to the fact that our studied area is larger in the North-South than in the EastWest direction, we do not expect a significant contribution from objects beyond the survey boundaries because the cloud is mostly covered by our observations (see e.g. Mizuno et al. 1999). Thus, we do not correct for boundary effects, which could introduce a new bias in the analysis given that such corrections are generally based on the assumption of a random distribution beyond the boundaries.
} 
Table 6. Infrared photometry for candidate low-mass members of Chamaeleon I ${ }^{a}$.

\begin{tabular}{|c|c|c|c|c|c|c|c|c|}
\hline Name & $J_{D}$ & $K s_{D}$ & $J$ & $H$ & $K$ & $L$ & $F_{6.7}(\mathrm{mJy})$ & $F_{14.3}(\mathrm{mJy})$ \\
\hline ChaI 403 & 13.28 & 10.83 & & & & & & \\
\hline ChaI 404 & 12.34 & 10.80 & & & & & $20.0 \pm 0.7$ & $5.5 \pm 1.2$ \\
\hline ChaI 405 & 12.34 & 10.80 & & & & & $20.0 \pm 0.7$ & $5.5 \pm 1.2$ \\
\hline ChaI 406 & 13.75 & 12.30 & & & & & $5.5 \pm 0.8$ & $6.8 \pm 1.2$ \\
\hline ChaI 410 & 14.07 & 13.02 & & & & & $15.3 \pm 0.8$ & $17.6 \pm 1.3$ \\
\hline ChaI 411 & 12.65 & 11.06 & 12.74 & 11.65 & 11.10 & 10.67 & $15.6 \pm 1.5$ & $16.5 \pm 1.5$ \\
\hline ChaI $412^{b}$ & 14.36 & 12.38 & 14.57 & 13.33 & 12.48 & & $5.3 \pm 0.7$ & $6.7 \pm 1.1$ \\
\hline ChaI 417 & 12.38 & 9.93 & 12.74 & 11.12 & 10.30 & 8.58 & $34.4 \pm 1.0$ & $35.1 \pm 1.4$ \\
\hline ChaI 418 & 12.19 & 10.42 & 12.48 & 11.12 & 10.48 & 9.55 & $14.9 \pm 1.3$ & $12.3 \pm 2.3$ \\
\hline ChaI 420 & 13.10 & 8.83 & 12.96 & 10.28 & 8.90 & 7.74 & $113.5 \pm 1.8$ & $36.2 \pm 1.4$ \\
\hline ChaI 422 & 13.05 & 11.91 & & & & & $3.2 \pm 0.7$ & \\
\hline ChaI 425 & 11.26 & 9.96 & & & & & $11.4 \pm 0.8$ & $3.7 \pm 1.0$ \\
\hline ChaI 427 & 13.40 & 10.01 & & & & & & \\
\hline ChaI 428 & 13.75 & 10.50 & & & & & & \\
\hline ChaI 429 & & & 13.62 & 12.47 & 11.83 & 10.72 & & \\
\hline ChaI 432 & 12.72 & 10.08 & & & & & $12.6 \pm 1.3$ & \\
\hline ChaI 433 & 12.78 & 10.51 & & & & & $7.0 \pm 1.1$ & \\
\hline ChaI 434 & 12.39 & 10.93 & 11.92 & 10.92 & 10.47 & 10.03 & $7.4 \pm 1.3$ & \\
\hline ChaI 438 & 13.01 & 11.56 & & & & & $7.6 \pm 1.2$ & $8.2 \pm 1.5$ \\
\hline ChaI 441 & 14.55 & 11.69 & & & & & $3.4 \pm 0.7$ & \\
\hline ChaI 448 & 11.30 & 10.14 & & & & & $11.2 \pm 0.8$ & \\
\hline ChaI 449 & 11.29 & 10.09 & & & & & $13.2 \pm 1.1$ & \\
\hline ChaI 450 & 10.94 & 9.27 & & & & & $20.7 \pm 0.8$ & \\
\hline ChaI 453 & 11.23 & 10.16 & & & & & $15.0 \pm 0.8$ & $7.5 \pm 1.9$ \\
\hline ChaI 456 & 11.41 & 10.36 & & & & & & \\
\hline ChaI 457 & 11.78 & 10.61 & & & & & & \\
\hline ChaI 603 & 12.10 & 10.27 & & & & & $23.1 \pm 1.4$ & $14.4 \pm 2.1$ \\
\hline ChaI 607 & 14.54 & 10.48 & 14.46 & 11.72 & 10.47 & 9.92 & $14.3 \pm 1.1$ & \\
\hline ChaI 608 & 11.74 & 9.04 & 11.71 & 9.86 & 9.08 & 8.45 & & \\
\hline ChaI 609 & 13.08 & 9.87 & 13.19 & 11.00 & 9.61 & 8.29 & & \\
\hline ChaI 610 & 12.31 & 11.13 & 12.46 & 11.57 & 11.10 & 10.38 & $4.8 \pm 1.2$ & \\
\hline ChaI 613 & 13.73 & 12.34 & & & & & $4.3 \pm 1.1$ & \\
\hline ChaI 615 & 11.79 & 10.77 & & & & & $5.9 \pm 0.7$ & \\
\hline ChaI 616 & 10.46 & 8.73 & 10.59 & 9.38 & 8.94 & 8.51 & $26.6 \pm 1.1$ & $6.7 \pm 1.2$ \\
\hline ChaI 622 & 10.93 & 9.46 & & & & & & \\
\hline ChaI 625 & 11.60 & 10.64 & & & & & & \\
\hline ChaI 708 & 11.64 & 10.67 & 11.81 & 11.34 & 10.79 & 10.28 & & \\
\hline ChaI 709 & 12.45 & 11.30 & 12.38 & 11.66 & 11.25 & 10.53 & $7.5 \pm 1.5$ & \\
\hline ChaI 721 & 11.10 & 9.26 & & & & & $24.0 \pm 1.9$ & \\
\hline ChaI 726 & 13.28 & 11.70 & 13.36 & 12.34 & 11.65 & 10.76 & $10.3 \pm 2.5$ & \\
\hline ChaI 731 & 13.13 & 9.32 & & & & & $383.1 \pm 5.6$ & $611.3 \pm 19.3$ \\
\hline ChaI 735 & 13.03 & 11.38 & 12.92 & 11.96 & 11.36 & 10.90 & $12.0 \pm 1.8$ & \\
\hline ChaI 742 & 11.69 & 10.66 & & & & & & \\
\hline ChaI 747 & 11.78 & 10.64 & & & & & & \\
\hline ChaI 748 & 11.53 & 10.45 & & & & & & \\
\hline ChaI 752 & 10.45 & 9.09 & 10.56 & 9.56 & 9.20 & 8.92 & $26.8 \pm 2.0$ & \\
\hline ChaI 760 & 10.77 & 8.51 & 10.87 & 9.34 & 8.55 & 7.69 & $186.3 \pm 6.2$ & $238.5 \pm 8.8$ \\
\hline
\end{tabular}

a $J_{D} K s_{D}$ : DENIS photometry (Cambrésy et al. 1998); JHKL: Photometry from Kenyon \& Gómez (2001).

${ }^{b}$ Photometry from Gómez \& Kenyon (2001).

be more clustered near HD 97048 (a Herbig Ae/Be star) than near HD 97300 (a likely zero-age main sequence A star). A third peak is observed in both cases at the distance corresponding to the boundaries of the third cloud core between both stars (at around 30' from HD 97048 and 40' of HD 97300). Although some of the objects in the bins could actually be very far away from this core, the peak is significant because it appears when counting from both stars. 


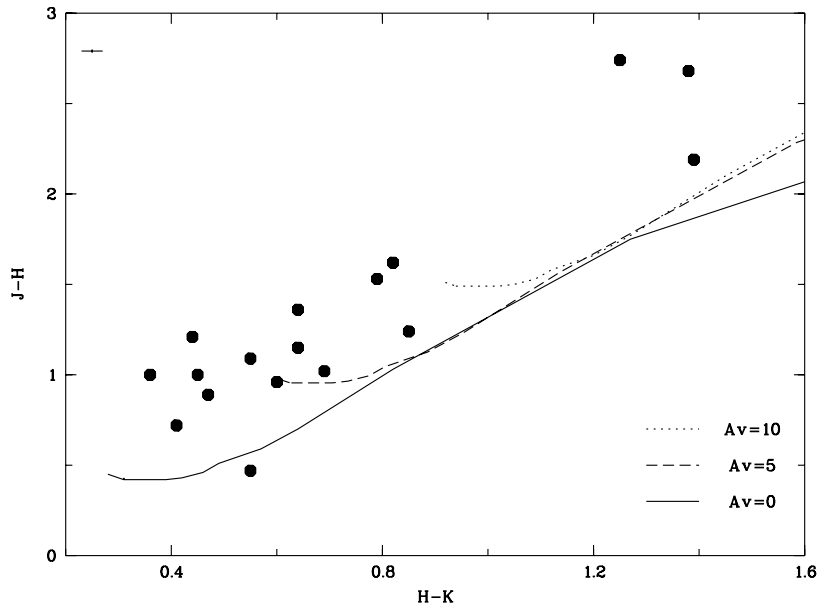

Fig. 9. Colour-colour diagram for our candidates with near-infrared photometry. The curves indicate the position of a $1 \mathrm{Myr}$ isochrone from Chabrier et al. (2000) for different extinction values. The crossed lines on the upper left corner of the plot indicate the average errors according to Kenyon \& Gómez (2001).

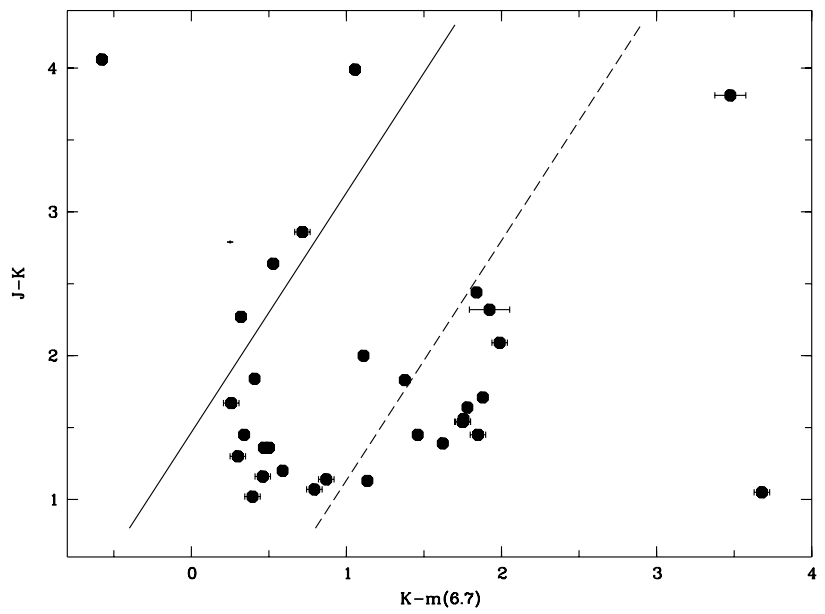

Fig. 10. $(J-K, K-m(6.7))$ colour-colour diagram for our candidates detected in the near and mid-infrared. The objects with intrinsic $(K-$ $m(6.7)$ ) excess are found to the right of the assumed reddening band, indicated by the two parallel lines.

\section{Binary objects}

To look for possible binary pairs among our objects, we adopt an upper separation cutoff of $12^{\prime \prime}$ (about $1800 \mathrm{AU}$ ). This value was determined by estimating the average surface density in our surveyed area. Then the distance was computed at which a given object should have a single neighbour if there were a uniform distribution on the whole field; this distance turned out to be about 12 '. 2 . Previous studies of visual binaries in Chamaeleon I (e.g. Reipurth \& Zinnecker 1993; Brandner et al. 1996) also used an upper limit of $12^{\prime \prime}$. Indeed, inspection of our images shows that the probability of finding a neighbouring object is notably increased at a slightly larger separation.

Most of our objects are found in isolation, or at least do not have neighbours down to the distances resolvable from our images. In very few cases one of our objects is found near a previously known cloud member: ChaI 625 , classified as a transition
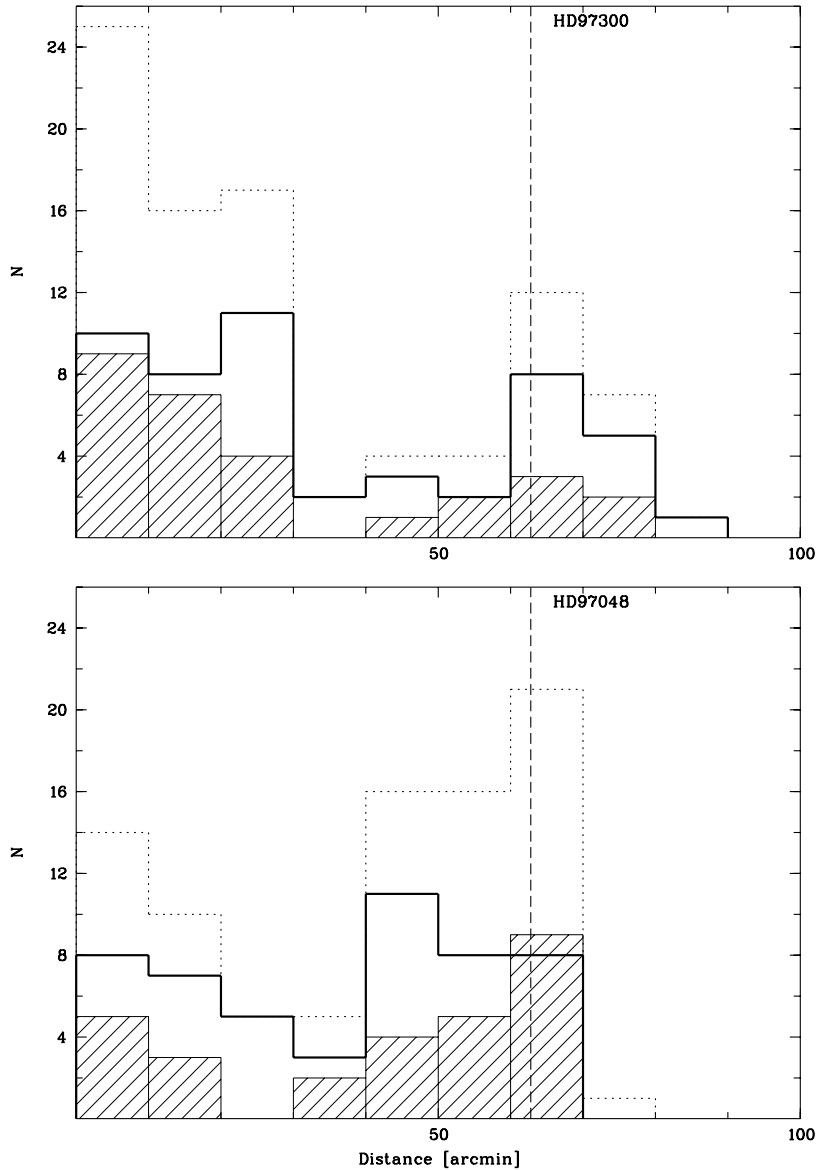

Fig. 11. Distribution of the low-mass Cha I members according to the distance to the intermediate mass stars HD 97048 (upper panel) and HD 97300 (lower panel). The blank histograms show the distribution of the very low-mass stars; the hashed histograms, of the brown dwarfs and faint brown dwarf candidates. The general histograms for all these objects are showed in dotted lines. The distance to the other intermediate mass star is also indicated.

object (spectral type M 6), is identified as the brightest component of the visual binary Hn12 (Schwartz 1991). The M 4 star ChaI 408 is located at about 14" of the reported position for the star Sz 37 (which lies beyond our survey boundaries), and is probably not physically bound to it.

As mentioned in the previous section, the pair formed by ChaI 404 and ChaI 405 lie whithin a separation of $\sim 9 . ' 5$. If it were a true cloud member, ChaI 404 would be a brown dwarf candidate with an estimated spectral type of M 6.5. ChaI 405 has spectral type M 5.5 and is most probably a very low-mass star. This system could still have a third member at about $5^{\prime \prime}$ ( 750 AU) of ChaI 404. This object is only visible in our longest I exposure, with a measured magnitude of $I \simeq 19$ mag. Considering our detection limits, it thus seems to have a very red colour, $R-I \gtrsim 3$, which would fit the empirical isochrone for the Chamaeleon members shown in Fig. 2. If this faint object indeed belonged to the cloud, it could be a very low-mass companion (a very low-mass brown dwarf or a giant planet) of this possible binary system. We show this possible multiple system in Fig. 12. 


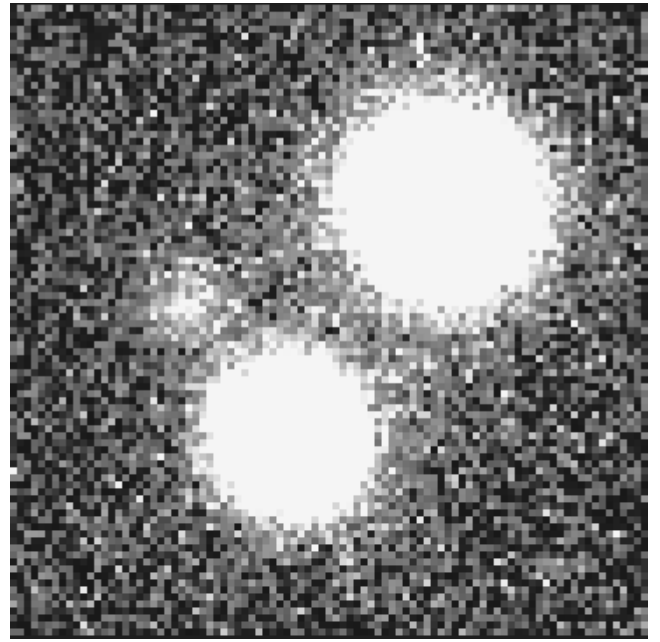

Fig. 12. Detail of the long $I$ exposure showing the possible multiple system formed by the star ChaI 405 (upper object), the brown dwarf candidate ChaI 404 (lower object) and a very faint object towards the north-east of ChaI 404. The two brightest components lie at a separation of about 9.5. This system is found at the position of the ISOCAM source ISO 250. (North is up and east is left in this image.)

Another wide pair with a separation of $\sim 10^{\prime \prime}$ is formed by ChaI 608 and ChaI 609. The latter is a star of spectral type M 5 according to our classification. Its location corresponds to the infrared-detected star Baud 38 (Baud et al. 1984). On the other hand, the position of the ROSAT X-ray source CHXR 30 (Feigelson et al. 1993) is well matched with that of ChaI 608. As mentioned in Sect. 3.1, this object had a slightly too blue $(R-I)$ colour to be included in our original candidate selection. We have found only one previous reference for a double object in a recent paper (Carpenter et al. 2002), though already Feigelson et al. reported an offset of 7 " between the positions of the infrared and the X-ray sources. However, in our own inspection of the ROSAT data (López Martí et al. 2002), we do find a bright elongated source at the position of this binary candidate, meaning that the X-ray source could actually be double and include both ChaI 608 and ChaI 609.

The pair formed by ChaI 735 and ChaI 737 (spectral types M 5.5 and M 8, respectively), if actually bound, would be a good candidate for a wide star-brown dwarf system. However, these objects lie at a separation of $\sim 14$.' 5 , so they have a high probability of not being physically connected.

We also checked our and the previously known objects for faint or very close companions that could have been missed by the automatic object search. We only find a possible companion near a brown dwarf candidate, ChaI 425 (spectral type M 7.5), only visible in the longest I exposure as a faint "tail" to this object. It was not possible to get the photometry of this possible companion from the subtracted image, due to the residuals left by the subtraction of the brightest object (which is almost saturated in the longest exposure). On the other hand, as explained in Sect. 3.2.3, ChaI 456 is probably an unresolved stellar binary. ChaI 726 (a brown dwarf candidate of spectral type M 8.5) seems to be slightly elongated in the north-south direction and could be a very close binary as well ${ }^{7}$.

Although in several cases faint objects are seen near our identified cloud members, their $(R-I)$ colours (when both $I$ and $R$ photometry are available) seem to indicate that they belong to the background. Also some of the previously known brown dwarf candidates have faint neighbours visible in our images. These objects have recently been studied by Neuhäuser et al. (2002), who conclude from their infrared colours that they are unlikely to belong to the Chamaeleon I cloud, and thus to be bound to the brown dwarf candidates. To confirm if all these objects have close companions, spectroscopic observations as well as more imaging observations are needed.

From this analysis we conclude that most of our objects are not members of (wide) binary systems. Hence, binarity (down to the separations that we can prove) should not significantly change the Chamaeleon I mass function.

\section{Accretion processes}

\section{1. $\mathrm{H} \alpha$ emission}

The detection of $\mathrm{H} \alpha$ emission in young objects is commonly regarded as indicative of an ongoing accretion process, and/or a measure of chromospheric activity. As explained in Sect. 3.1, there is a relation between the $\mathrm{H} \alpha$ equivalent width $E W(\mathrm{H} \alpha)$ and the $(\mathrm{H} \alpha-R)$ colour index for the objects studied by CNK00 (Fig. 3). Since our new very low-mass stars and brown dwarfs have similar $(\mathrm{H} \alpha-R)$ colours, they should also have similar $\mathrm{H} \alpha$ equivalent widths, with values ranging between 10 and $30 \AA$ A. In TTS, such values are indicative of a significative accretion process.

In Fig. 3 there is one remarkable outlayer: Cha $\mathrm{H} \alpha 1$. This bona-fide brown dwarf should have an equivalent width of $\sim 20 \AA$ according to its $(\mathrm{H} \alpha-R)$ colour. However, it has a reported equivalent width between 34 and $99 \AA$ (CRN99; Neuhäuser \& Comerón 1999; CNK00 - the plotted data), being by far the strongest (and most variable) emitter of the whole data set. Natta \& Testi (2001) showed that Cha $\mathrm{H} \alpha 1$ is surrounded by a disk with similar characteristics to the ones around the more massive TTS. However, they also report a disk around $\mathrm{Cha} \mathrm{H} \alpha 2$ and $\mathrm{Cha \textrm {H }} \alpha$ 9, whose $\mathrm{H} \alpha$ emission does not show a strong deviation from the general correlation in Fig. 3. On the other hand, Cha $\mathrm{H} \alpha 1$ also has strong X-ray emission (Neuhäuser \& Comerón 1998), probably an indicator of coronal magnetic activity. Therefore, its "abnormal" $\mathrm{H} \alpha$ emission could also be related to magnetic (chromospheric) activity, or be the result of the combination of both accretion and chromospheric activity. A distinction between the two processes possibly leading to the strong $\mathrm{H} \alpha$ emission, however, cannot be made with the available data.

Recent observations show that some objects with photometric variability are characterized by a large $\mathrm{H} \alpha$ equivalent width (Eislöffel \& Scholz 2002). However, no

\footnotetext{
Recalling the discussion from Sect. 3.2.3, if this were the case, the individual components of this binary system would probably have earlier spectral types than estimated from our study, and they might not be brown dwarfs.
} 


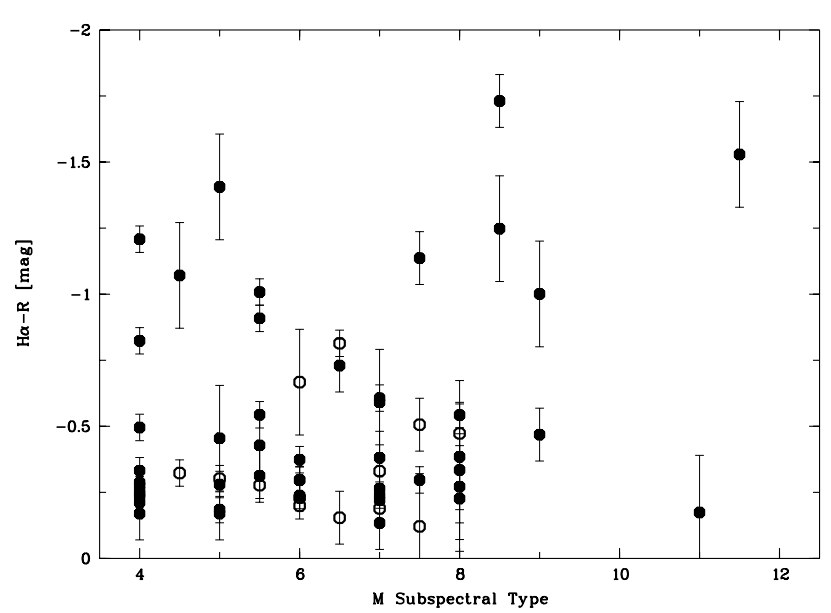

Fig. 13. Measured $(\mathrm{H} \alpha-R)$ colour index versus spectral type. Symbols as in Fig. 2. The strongest emitters are among the objects with the latest spectral types.

photometric variability of $\mathrm{ChaH} \alpha 1$ has been reported so far, although observations from different epochs are available (Neuhäuser \& Comerón 1999; CRN99; CNK00; Neuhäuser et al. 2002). A study of the variability of some previously known brown dwarfs in Chamaeleon I was recently performed by Joergens et al. (2003), but these authors did not consider Cha $\mathrm{H} \alpha 1$ due to its poor $S / N$ in their images. On the other hand, they find quite long photometric periods for ChaI $\mathrm{H} \alpha 2$ and ChaI $\mathrm{H} \alpha 3$ (3.2 and 2.2 days, respectively), while no variability was detected for ChaI $\mathrm{H} \alpha 4,5,8$ and 12. All these objects are included in Fig. 3. Since Cha $\mathrm{H} \alpha 1$ is the only one showing a different behaviour in its $\mathrm{H} \alpha$ emission, it seems unlikely that it is connected to (long) photometric variability.

Figure 13 shows the measured $(\mathrm{H} \alpha-R)$ colours for all our objects with estimated spectral type. Although no clear systematic behaviour is observed, interestingly, many of the objects with $(\mathrm{H} \alpha-R)$ colours smaller than -1 (what roughly corresponds to equivalent widths greater than $30 \AA$ ) have spectral types later than M7. Thus, they are probably brown dwarfs. Despite the larger photometric errors towards later spectral types, i.e. fainter objects, it is remarkable that almost all the objects with spectral type later than about M 8 are found to have such extreme $(\mathrm{H} \alpha-R)$ colours. However, the low number of objects does not allow us to state firmly whether this is a typical property of substellar objects. Given that they are just a small subset of our sample (14\%), it may be that these objects were observed near a maximum of activity. It should be noted that this result may simply be a consequence of the sensitivity limit of our survey. Low luminosity objects showing moderate to low $\mathrm{H} \alpha$ emission may appear below the completeness magnitude and close to the detection limit of the $\mathrm{H} \alpha$ images, while on the contrary objects with strong emission are detected more easily.

Very strong $\mathrm{H} \alpha$ emission has also been reported for a brown dwarf candidate in Corona Australis (Fernández \& Comerón 2001). This object, LS-RCrA 1 (M 6.5), displays a very particular spectrum with spectral features of accretion and mass loss. This could also be the case of our strong $\mathrm{H} \alpha$ emitters, as they probably have disks (see Sect. 6.2 below). Such a result would be surprising because, if brown dwarfs form from the collapse of a molecular cloud core, they are expected to have very low accretion rates. Otherwise, they would eventually accrete enough mass to start the hydrogen burning process. This is in contradiction with the observation of strong $\mathrm{H} \alpha$ emission. We note, however, that this emission can seem stronger in latetype objects simply due to a lower continuum. Moreover, indications of accretion have not been found in all substellar objects showing a prominent $\mathrm{H} \alpha$ emission line. This is the case e.g. for S Ori 71, a substellar candidate member of the $\sigma$ Orionis cluster (Barrado y Navascués et al. 2002). These authors discuss other possible causes of the $\mathrm{H} \alpha$ emission, such as chromospheric activity or mass exchange between the components of a binary system. Complementary spectroscopic observations of our objects will certainly help to clarify the origin and characteristics of their $\mathrm{H} \alpha$ emission.

We also analysed the behaviour of the $\mathrm{H} \alpha$ flux with the spectral type. Since no standards were available for the $\mathrm{H} \alpha$ filter, we computed a "pseudoflux" using the relation:

$\tilde{F}_{\mathrm{H} \alpha}=F_{\mathrm{H} \alpha} / F_{0}=10^{-m_{\mathrm{H} \alpha} / 2.5}$,

where $m_{\mathrm{H} \alpha}$ is the measured magnitude in the $\mathrm{H} \alpha$ filter. The difference between the logarithm of the pseudoflux $\tilde{F}_{\mathrm{H} \alpha}$ and that of a hypothetical absolute flux $F_{\mathrm{H} \alpha}$ is then a constant value, $\log F_{0}$, depending on the reference photometric system.

The upper panel in Fig. 14 shows $\log \tilde{F}_{\mathrm{H} \alpha}$ versus $M$ subspectral type for Chamaeleon I. A roughly linear decrease of the flux with later spectral type is clearly seen, consistent with the progressive faintness of the objects. There is no indication for a change at the stellar/substellar boundary. The apparent saturation for the latest spectral types is most probably due to the sensitivity limit of our $\mathrm{H} \alpha$ survey.

A decrease of the chromospheric activity with the spectral type has been reported by Gizis et al. (2000) for older M- and L-type dwarfs. Also Zapatero Osorio et al. (2002a) found a similar behaviour in a sample of very low-mass members in the young $\sigma$ Orionis cluster. We note, however, that these authors used $L_{\mathrm{H} \alpha} / L_{\mathrm{bol}}$ instead of the $\mathrm{H} \alpha$ flux in their study, and thus our result is not directly comparable with theirs. $L_{\mathrm{H} \alpha} / L_{\mathrm{bol}}$ is probably a better activity indicator, since it does not depend on the distance or the radius of the objects.

The lower panel in Fig. 14 is a plot of $\log \tilde{L} x_{\mathrm{H} \alpha} / L_{\mathrm{bol}}$ versus the spectral type for our young objects in Chamaeleon I. To obtain the bolometric luminosities, we have proceeded as in CNK00, using $(R-I)$ intrinsic colours from Kenyon \& Hartmann (1995) and Zapatero Osorio et al. (1997). Interestingly, we do not see a decrease in the $\mathrm{H} \alpha$ emission in these data. The situation reminds us of the results of Mokler \& Stelzer (2002), who studied the activity of a large sample of young very low-mass objects and found no decrease in $L_{\mathrm{x}} / L_{\mathrm{bol}}$ down to spectral type M 7, although a decrease in $L_{\mathrm{x}}$ was clearly seen in their data.

Zapatero Osorio et al. (2002a) noted that young objects displayed, on average, higher $\mathrm{H} \alpha$ emission than their older field counterparts, the difference being higher for L-type objects. They explained this result as a consequence of the decline of magnetic activity and/or mass accretion with age. Such a direct comparison between the $\mathrm{H} \alpha$ luminosities is not possible with 

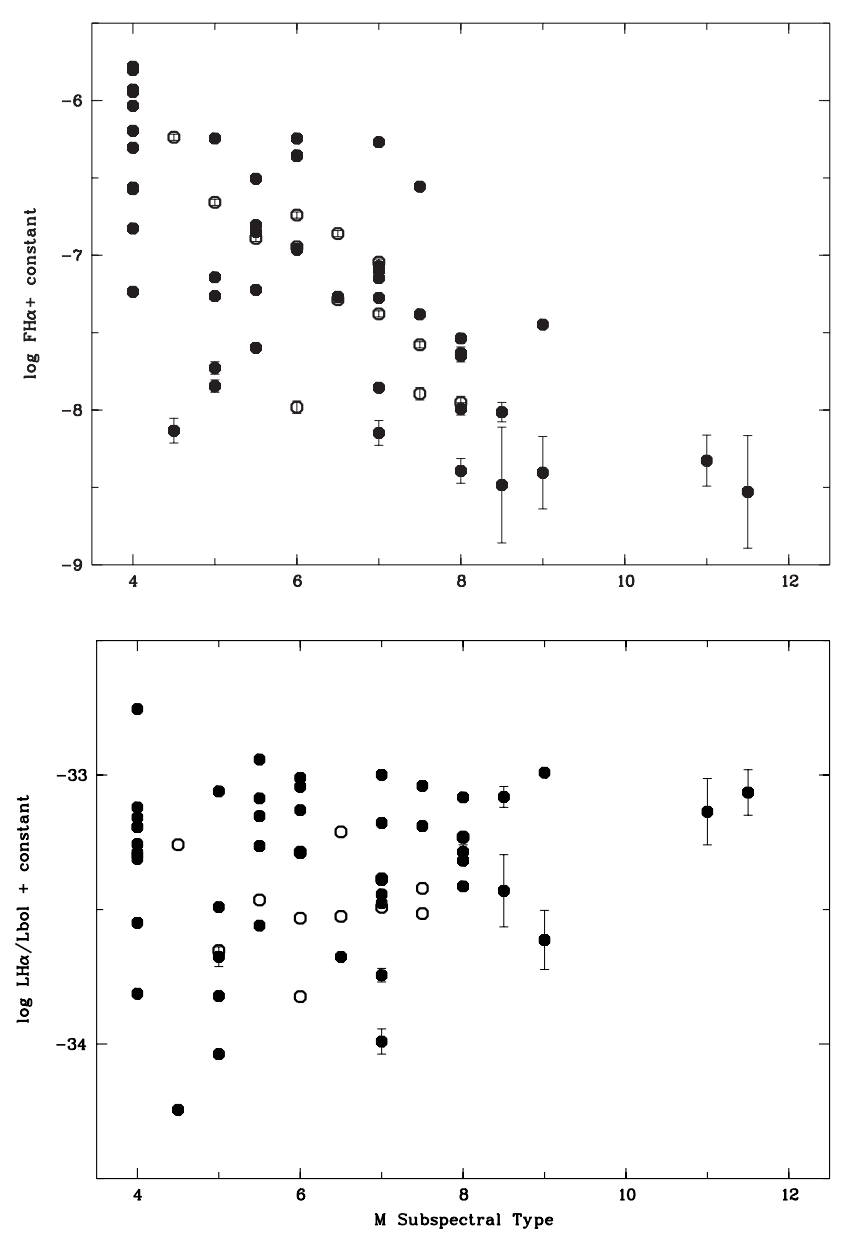

Fig. 14. Upper panel: Measured $\mathrm{H} \alpha$ pseudoflux versus spectral type for our objects in Chamaeleon I. Symbols as in Fig. 2. The flux clearly decreases with later spectral type. Lower panel: $\log L_{\mathrm{H} \alpha} / L_{\mathrm{bol}}$ versus spectral type for our objects in Chamaeleon I.

our data, however, because of the lack of an absolute $\mathrm{H} \alpha$ photometric system.

\subsection{Evidence for disks}

Figure 15 is a plot of the colour index defined by the two ISOCAM passbands, $m(6.7)-m(14.3)$ (computed with Eqs. (6) and (7)), versus our measured $(\mathrm{H} \alpha-R)$ colour index. All our objects with strong $\mathrm{H} \alpha$ emission from their $(\mathrm{H} \alpha-R)$ colours are found to have mid-infrared excess according to Persi et al. (2000). The detection of such an excess from young stellar objects is a well-known observational signature of a circumstellar disk. Hence, $\mathrm{H} \alpha$ emission is related to the presence of an accretion disk around these objects.

A similar proportion of the stars and brown dwarfs in our sample (around 30\% and 37\%, respectively) have a reported mid-infrared excess. On the other hand, while around $52 \%$ of the stars and $67 \%$ of the transition objects show nearinfrared excess, only about $37 \%$ of the brown dwarfs do. Interestingly, mid- and near-infrared excess do not seem to be correlated: of the brown dwarfs with mid-infrared excess, just around $10 \%$ show also near-infrared excess. This percentage slightly increases for transition objects and low-mass

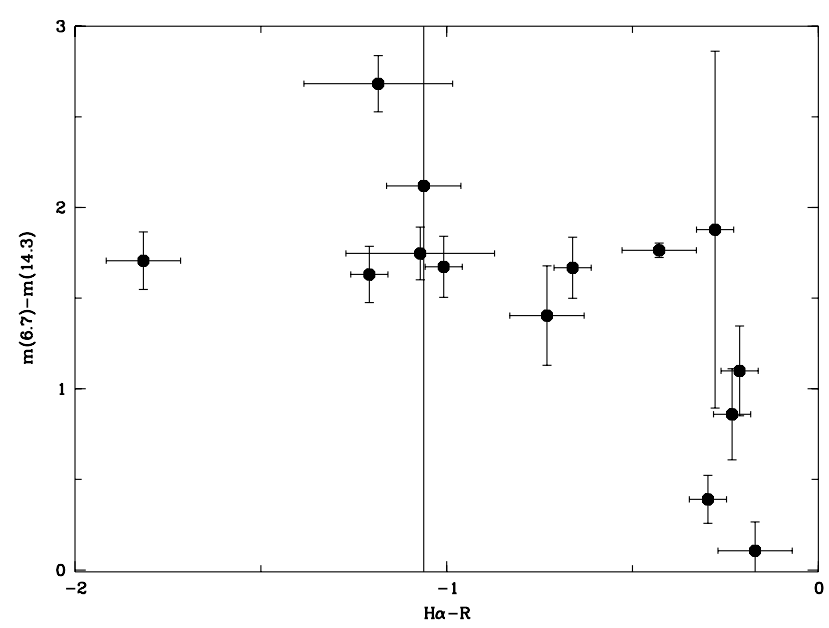

Fig. 15. ( $\mathrm{H} \alpha-R, m(6.7)-m(14.3))$ colour-colour diagram for the objects in our sample detected by ISOCAM in both passbands. The objects with very strong $\mathrm{H} \alpha$ emission have mid-infrared excess.

stars (17\% and $21 \%$, respectively). However, the total proportion of objects detected with both kinds of excesses is only around $13 \%$. This is in good agreement with the results of CNK00, who found that several of the previously known brown dwarfs and brown dwarf candidates in Chamaeleon I (selected from an $\mathrm{H} \alpha$ survey) showed mid-infrared excess, in spite of lacking near-infrared excesses.

For TTS, the lack of near-infrared excess is usually attributed to an inner hole in the circumstellar disk. However, strong $\mathrm{H} \alpha$ emission is normally interpreted as a signature of the accretion process onto the surface of the object, what requires the presence of an inner disk. In a recent paper, Natta $\&$ Testi (2001) show that the observed spectral energy distribution of three of the previously known brown dwarfs and brown dwarf candidates in Chamaeleon I can be explained if they are surrounded by accretion disks. They remark that, in the case of brown dwarfs, the presence of disks is difficult to infer from near-infrared photometry alone, where the emission is largely dominated by the photosphere. Also Oliveira et al. (2002), who performed infrared observations of a sample of very lowmass objects in the $\sigma$ Orionis cluster, conclude that only a very small proportion of them show significant near-infrared excess. Despite this negative result, some of the low-mass objects in this cluster show $\mathrm{H} \alpha$ emission and other spectral features characteristic of an accretion process (Zapatero-Osorio et al. 2002 b). Indeed, a recent $L$-band study has increased the disk frequency of the low-mass $\sigma$ Orionis members up to about $50 \%$, remarkably larger than inferred from the $K$-band excess alone (Oliveira et al. 2003).

In any case, the indications for the presence of disks around many of our brown dwarfs and brown dwarf candidates favours the hypothesis that they formed in a similar way as TTS, and leave open the possibility of brown dwarfs forming planetary systems.

Although mid-infrared photometry is available for two of our faintest objects (ChaI 607 and ChaI 441), neither of them shows an excess according to the ISOCAM observations. However, ChaI 607, and also ChaI 428, have a near-infrared 


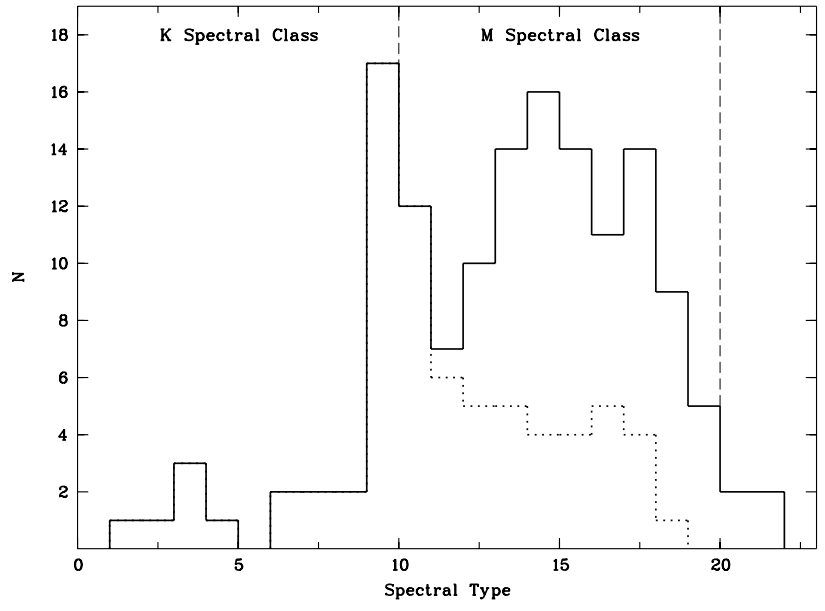

Fig. 16. Histogram showing the distribution of spectral types for the identified low-mass stars and brown dwarfs in Chamaeleon I. The dotted line is the distribution for the objects studied by previous authors (Gauvin \& Strom 1992; Lawson et al. 1996; CRN99 and CNK00). The local minimum at M 2-M 3 may be due to the incompleteness of our sample in this range (see text).

excess. As argued in Sects. 3.2.3 and 3.3, both ChaI 607 and ChaI 428 may be very reddened stars. Their observed excess would then be due to their high extinction (with values in the $J$ band of 4.9 and 3.9, respectively, according to Gómez \& Mardones 2003). It seems thus probable that also ChaI 441 is an extincted star, rather than a low-mass brown dwarf.

\section{The mass function in Chamaeleon I}

The task of constructing the substellar initial mass function is not an easy one because the theoretical models still present large uncertainties for such very young objects $(<10 \mathrm{Myr})$. Especially relevant is the choice of initial conditions: The picture of non-accreting objects contracting from large initial radii, as used in most of the models, is an idealized description, which can drastically affect the fundamental properties (luminosity and effective temperature) of objects younger than about 10 Myr (Baraffe et al. 2002). From the observational point of view, the main problems are the uncertainties in the luminosities and spectral types for these young, often highly extincted objects. Moreover, recent observations show that very young brown dwarfs can be variable (e.g. Eislöffel \& Scholz 2002). Therefore, any attempt to infer an age or a mass from observable quantities for these objects must be considered with caution.

On the other hand, at these very early stages of evolution there is a good correlation between the spectral type (i.e. the effective temperature) and the mass. Thus, as a first step, we can study the spectral type distribution of the Chamaeleon I members shown in Fig. 16. The histogram includes all the new members with a determined spectral type from Table 4 (except the few dubious objects discussed in the previous sections), as well as the objects in the surveyed area with identified spectral type from Gauvin \& Strom (1992), Lawson et al. (1996), CRN99 and CNK00. The dotted line indicates the histogram for these previously known stars. Only the objects with spectral types between $\mathrm{K} 0$ and L 2 are shown. A numeric code is used: the numbers 2 to 9 correspond to $\mathrm{K} 0$ to $\mathrm{K} 7$ objects, respectively; 10-19 indicate spectral types from M 0 to M9; and numbers later than 20 are asigned to the L-type objects. Few objects of spectral types earlier than $\mathrm{K}$ are known in the cloud: Gauvin \& Strom report four objects of spectral type G, as well as the intermediate-mass stars HD 97048 (B9) and HD 97300 (A0).

Two peaks are seen in the spectral type distribution. The first one corresponds to late K objects. Nonetheles, the decrease in the number of objects for the spectral types M 0-M 3 might not be a real characteristic of the region, but a consequence of the incompleteness of our sample in this range. We recall that the (M 855-M915) colour index saturates for spectral types earlier than M4; hence, twelve objects in our survey that have a spectral type earlier than M4 but lack a previous spectroscopic classification are not included in Fig. 16. The second peak is seen for spectral types around M 5, approximately at the star/brown dwarf transition, indicating a decrease of the number of objects in the substellar regime. Taking M 5 as the stellar/substellar boundary, we count around 100 stars (including the ones from our study whose spectral type is unknown) and about 51 brown dwarfs and brown dwarf candidates. Hence, the number of stars in the surveyed area is roughly doubling that of brown dwarfs. It must be noted, however, that this is just a lower limit to the actual number of brown dwarfs in the cloud, because some low-mass objects without (detectable) $\mathrm{H} \alpha$ emission will be missed due to our selection criteria. Moreover, extincted brown dwarfs are more difficult to detect than stars, especially at optical wavelengths.

Taking into account the uncertainties in our absolute photometry and spectral types (recall discussions in Sects. 2.3 and 3.2.3), especially for objects earlier than M4, we can now construct the mass function in Chamaeleon and give an estimation for the value of the exponent $\alpha$ of the usual approximation for the mass function:

$\mathrm{d} N / \mathrm{d} M \sim M^{-\alpha}$.

For this calculation, we considered all known members between spectral types K 7 and M 7, for which our survey should be complete. According to the models of Baraffe et al. (1998), this range should correspond to masses of 1.2 to $0.025 M_{\odot}$ at the age of 1 Myr. We splitted the objects into two bins of stars (spectral types K 7-M 4, with masses of about 1.2-0.075 $M_{\odot}$ ) and substellar objects (spectral types M 5-M 7, with masses of about $0.075-0.025 M_{\odot}$ ). We did not take smaller bins because of the uncertainty in the classification for some of our objects with spectral types earlier than M4. By selecting two bins, we can include all the identified Chamaeleon I members in the calculation, even though we do not know their spectral types very accurately. There are 88 objects in the first and 39 in the second $\operatorname{bin}^{8}$. With these data, we derive an exponent of the mass function of

$\alpha=0.6 \pm 0.1$.

As mentioned above, some cloud members may have been missed because they do not have (measurable) $\mathrm{H} \alpha$ emission.

\footnotetext{
${ }^{8}$ We recall that the twelve objects with uncertain spectral type are not plotted in Fig. 16.
} 
To account for this possibility, we repeated the calculation several times including all the candidates from our $R$ and $I$ photometry that would belong to the first, second and both bins if they were indeed cloud members. These considerations lead us to the quoted error of 0.1 for the index of the mass function.

Our obtained index agrees surprisingly well with the value obtained by Moraux et al. (2003) for the Pleiades cluster $(0.60 \pm 0.11)$. As these authors point out, a similar value has been derived also for other clusters of similar age, thus older than Chamaeleon I. These results are also consistent with the values of $0.8 \pm 0.4$ and $0.7 \pm 0.2$ which Béjar et al. (2001) and Tej et al. (2002) found for the similarly old clusters $\sigma$ Orionis and IC 348, respectively. Hence, the shape of the IMF at the substellar boundary seems to be similar in all these young regions, regardless of their age and crowdedness.

A previous estimate of the index of the substellar IMF in Chamaeleon I had been done by CNK00. They obtained a value $\alpha=1.1$, larger than ours, implying a steeper increase of the mass function towards lower masses. The discrepancy might be due to the low number of objects in their study. We recall that the area covered by CNK00 is just $15 \%$ of our whole survey, and that they studied a region particularly dense in very lowmass objects.

\section{Constraints on formation models}

The spatial distribution of the brown dwarfs in Chamaeleon I is found to be a natural continuation of the T Tauri stars towards lower masses (see Sect. 4). Stellar and substellar objects also appear to have similar $\mathrm{H} \alpha$ emission properties (Sect. 6.1). These results, together with the presence of accretion disks around some brown dwarfs (Sect. 6.2) and the low number of possible star-brown dwarf systems (Sect. 5), lead us to assume that they most probably formed in the same way as TTS (that is, from the gravitational collapse of a molecular cloud) rather than in a circumstellar disk like planets do.

Moreover, the fact that the low-mass objects tend to be concentrated in particular areas seems to indicate that they formed very near the places where we currently see them. We note that a star with a tangential velocity of $1 \mathrm{~km} \mathrm{~s}^{-1}$ would have moved 3 pc in $3 \mathrm{Myr}$, which corresponds to about 1.1 at the distance of Chamaeleon I. Thus, if most of the brown dwarfs had been stellar embryos ejected from the stellar systems where they formed with an initial velocity of $1 \mathrm{~km} \mathrm{~s}^{-1}$, at the age of Chamaeleon I we should see them randomly distributed over the whole surveyed region, which is not the case. The typical velocity of our observed young stars and brown dwarfs cannot be higher than $0.2-0.4 \mathrm{~km} \mathrm{~s}^{-1}$ at most. This value is also remarkably lower than the average ejection velocities of $2 \mathrm{~km} \mathrm{~s}^{-1}$ predicted by the simulations of Bate et al. (2003). Joergens \& Guenther (2001) report a radial velocity dispersion of $2 \mathrm{~km} \mathrm{~s}^{-1}$ for several previously known brown dwarf and brown dwarf candidates around HD 97048. They argue, however, that this value is significantly smaller than the radial velocity dispersion of the TTS in Chamaeleon I $\left(3.6 \mathrm{~km} \mathrm{~s}^{-1}\right)$. On the basis of these considerations, it seems very unlikely that the brown dwarfs in Chamaeleon I have been ejected from multiple systems.
From the observed spatial distribution one may think that the existence of low-mass objects in Chamaeleon I could be somehow connected with the presence of the two intermediate mass stars. Dissipation by stellar winds from a nearby hot massive star could prevent the further growth of a stellar core, which would then remain a failed star - a brown dwarf. This mechanism has been invoked recently to explain the high concentration of substellar objects in Orion (Lucas \& Roche 2000; Lucas et al. 2001). The stars in Chamaeleon I, however, are not so hot and massive as the ones in Orion.

HD 97048, the one exhibiting a higher concentration of very low-mass neighbours, is a Herbig Ae/Be star, an object characterized by its strong winds. But it is doubtful whether winds from Herbig Ae/Be stars are powerful enough to evaporate the envelopes of surrounding star forming cores. This seems even more unlikely to be currently happening in the case of HD 97300, which might have reached the zero-age main sequence (e.g. Jones et al. 1985). Indeed, according to Gauvin \& Strom (1992), observations suggest that this part of the cloud is in a more evolved stage than the one containing the other intermediate mass star. This is also supported by the fact that the cloud density, as inferred from CO measurements, is significantly lower in this core than in the other two (Mizuno et al. 1999).

Furthermore, another remarkable concentration of lowmass objects is seen at the boundaries of the third cloud core, which does not contain any intermediate mass star. This core has not been as well studied as the other two. It is possible that we just observe the objects located in the outer part of this core, and that objects deep inside it are too obscured to be detected by our survey. Deeper observations are needed to clarify this point. If embedded brown dwarfs were detected, it would certainly mean that environmental conditions other than the presence of more massive stars are responsible for the formation of the substellar objects in Chamaeleon I.

Finally, our results from Sect. 7 indicate that the mass function of Chamaeleon I is rising towards lower masses. Hence, no matter how the brown dwarfs actually formed, this mechanism seems to be very efficient in producing substellar objects. The similar index $\alpha$ obtained for the mass functions of several young clusters and star forming regions hints to a similar formation process in all these regions.

\section{Conclusions}

We performed a deep WFI survey of about $1.2 \square^{\circ}$ of the Chamaeleon I cloud to identify new very low-mass members of this star-forming region. Candidates were selected from $R$ and $I$ photometry and their youth was tested by means of their $\mathrm{H} \alpha-R$ colours. We showed that this colour is a useful estimator of the $\mathrm{H} \alpha$ emission rate.

We developed a method to classify mid- to late $\mathrm{M}$ and early L-type objects from photometric observations in the two intermediate-band filters M 855 and M915. The colour defined by these two passbands, (M 855-M915), is correlated with the depth of the TiO and VO spectral features in these objects. In this way, we could provide the first spectral classification for about $80 \%$ of the objects in our sample. 
From our results, we conclude that the Chamaeleon I dark cloud contains a substantial amount of very low-mass objects, with its spectral type distribution showing a peak at about the star/brown dwarf transition boundary. Both kinds of objects have a similar spatial distribution, being placed predominantly near the cloud cores. Such a distribution does not seem to be a consequence of the presence of the two intermediate-mass stars, HD 97048 and HD 97300, because a large number of cloud members are probably associated with a dense core devoid of optical objects. We derive an index $\alpha=0.6 \pm 0.1$ of the mass function in the range from 1.2 to $0.025 M_{\odot}$, very similar to the values obtained by other authors in other young clusters and star forming regions.

Both the observed spatial distribution and the scarce number of visual pairs among our objects seem to indicate that the brown dwarfs in Chamaeleon I formed in a way similar to low-mass stars, and that they were not ejected from their parental systems, at least with high escape velocities (greater than $0.2-0.4 \mathrm{~km} \mathrm{~s}^{-1}$ ). Moreover, the detection of $\mathrm{H} \alpha$ emission connected to mid-infrared excess shows that at least some brown dwarfs probably have accretion disks, providing a further argument in favour of a star-like formation. On the other hand, near-infrared excess does not appear as a good diagnostic tool to the presence of disks around very low-mass objects.

The $\mathrm{H} \alpha$ emitting properties of the stars and brown dwarfs are very similar. There is no evidence for a change across the substellar transition. Contrary to other authors, we do not see a decrease in $\log L_{\mathrm{H} \alpha} / L_{\mathrm{bol}}$ for spectral types M4-M 9, meaning that the latest-type objects in this range could have at least as strong $\mathrm{H} \alpha$ emission as earlier-type objects. All this hints to a common origin for the emission in both stars and brown dwarfs, probably accretion processes, since both kinds of objects have disks. If it is confirmed, this result would contradict the commonly accepted picture that objects with lower mass should have lower accretion rates (hence, weaker $\mathrm{H} \alpha$ emission) than those with higher masses. However, with the available data we cannot exclude that other mechanisms, such as chromospheric activity, are (at least partially) responsible for this emission. In this context, it is interesting that a similar result has been obtained by Mokler \& Stelzer (2002) for the X-ray emission of substellar objects.

Acknowledgements. We are very grateful to H. Jahreiss for providing us with a list of objects from the Gliese-Jahreiss catalogue to be used as spectral standards; and to I. Baraffe, who calculated the mediumband filter isochrones for us. We kindly thank C. A. L. Bailer-Jones for his help with the field selection, P. Persi for the complete ISOCAM list, and K. Tachihara for the information about the CO data. We also acknowledge the useful suggestions made by R. Neuhäuser and by an anonymous referee, as well as many valuable comments from F. Comerón, A. Fernández Soto, J. Fabregat, J. M. Torrelles and J. M. Marcaide. J. E. thanks E. Pompei and the 2.2-m team for their help with the observations and the taxi services for their walkingimpaired visiting astronomer.

We made use of the SIMBAD database, operated at the Centre de Données astronomiques de Strasbourg (CDS) in Strasbourg (France). This work was supported by the German Deutsche Forschungsgemeinschaft, (DFG), projects numbers EI 409/7-1 and EI 409/7-2. B. L. M. also thanks ESO for financial support through its Director General's
Discretionary Funds during her stay at the ESO Headquarters in Garching.

\section{References}

Alcalá, J. M., Radovich, M., Silvotti, R., et al. 2002, SPIE, 4836, 406A

Appenzeller, I., \& Mundt, R. 1989, A\&ARv, 1, 291

Armitage, P. J., \& Bonnell, I. A. 2002, MNRAS, 330, L11

Baade, D., Meisenheimer, K., Iwert, O., et al. 1998, The ESO Messenger, 93, 13

Baraffe, I., Chabrier, G., Allard, F., \& Hauschildt, P. H. 1998, A\&A, 337, 403

Baraffe, I., Chabrier, G., Allard, F., \& Hauschildt, P. H. 2002, A\&A, 382,563

Barrado y Navascués, D., Stauffer, J. R., Briceño, C., et al. 2001, ApJS, 134,103

Barrado y Navascués, D., Zapatero Osorio, M. R., Martín, E. L., et al. 2002, A\&A, 393, L85

Bate, M. R., Bonnell, I. A., \& Bromm, V. 2003, MNRAS, 339, 577

Baud, B., Beintema, D. A., Wesselius, P. R., et al. 1984, ApJ, 278, L53

Béjar, V. J. S., Zapatero Osorio, M. R., \& Rebolo, R. 1999, ApJ, 521, 671

Béjar, V. J. S., Martín, E. L., Zapatero Osorio, M. R., et al. 2001, ApJ, 556,830

Bertin, E., \& Arnouts, S. 1996, A\&AS, 117, 393

Bouvier, J., Stauffer, J. R., Martín, E. L., et al. 1998, A\&A, 336, 490

Brandner, W., Alcalá, J. M., Kunkel, M., et al. 1996, A\&A, 307, 121

Briceño, C., Hartmann, L., Stauffer, J., \& Martín, E. 1998, AJ, 115, 2074

Cambrésy, L., Copet, E., Epchtein, N., et al. 1998, A\&A, 338, 977

Carpenter, J. M., Hillenbrand, L. A., Skrutskie, M. F., \& Meyer, M. R. 2002, AJ, 124, 1001

Chabrier, G., Baraffe, I., Allard, F., \& Hauschildt, P. 2000, ApJ, 542, 464

Comerón, F., Rieke, G. H., \& Neuhäuser, R. 1999, A\&A, 343, 477 (CRN99)

Comerón, F., Neuhäuser, R., \& Kaas, A. A. 2000, A\&A, 359, 269 (CNK00)

Delfosse, X., Tinney, C. G., Forveille, T., et al. 1999, A\&AS, 135, 41

Eislöffel, J., \& Scholz, A. 2002, AG Abstract Series, 19, A15

Eislöffel, J., Scholz, A., Mundt, R., et al. 2003, in preparation

Epchtein, N., de Batz, B., Capoani, L., et al. 1997, The ESO Messenger, 87, 27

Feigelson, E. D., Casanova, S., Montmerle, T., \& Guibert, J. 1993 , ApJ, 416, 623

Feigelson, E. D., \& Kriss, G. A. 1989, ApJ, 338, 262

Fernández, M., \& Comerón, F. 2001, A\&A, 380, 264

Gauvin, L. S., \& Strom, K. M. 1992, ApJ, 385, 217

Gizis, J. E., Monet, D. G., Reid, I. N., et al. 2000, ApJ, 120, 1085

Gómez, M., \& Kenyon, S. J. 2001, AJ, 121, 974

Gómez, M., \& Persi, P. 2002, A\&A, 389, 494

Gómez, M., \& Mardones, D. 2003, AJ, 125, 2134

Gregorio-Hetem, J. C., Sanzovo, G. C., \& Lepine, J. R. D. 1989, A\&AS, 79, 452

Hartigan, P. 1992, BAAS, 24, 1262

Joergens, V., \& Guenther, E. 2001, A\&A, 379, L9

Joergens, V., Fernández, M., Carpenter, J. M., \& Neuhäuser, R. 2003, ApJ, 594, 971

Jones, T. J., Hyland, A. R., Harvey, P. M., et al. 1985, AJ, 90, 1191

Kenyon, S. J., \& Gómez, M. 2001, AJ, 121, 2673

Kenyon, S. J., \& Hartmann, L. 1995, ApJS, 101, 117

Kirkpatrick, J. D., Henry, T. J., \& McCarthy, D. W. 1991, ApJS, 77, 417 
Kirkpatrick, J. D., Reid, I. N., Liebert, J., et al. 1999, ApJ, 519, 802

Kirkpatrick, J. D., Reid, I. N., Liebert, J., et al. 2000, AJ, 120, 447

Lamm, M., Bailer-Jones, C. A. L., Mundt, R., et al. 2004, A\&A, in press

Landolt, A. U. 1992, AJ, 104, 340

Lawson, W. A., Feigelson, E. D., \& Huenemoerder, D. P. 1996, MNRAS, 280, 1071

López Martí, B., Stelzer, B., \& Neuhäuser, R. 2002, AG Abstracts Series, 19, P80

Lucas, P. W., \& Roche, P. F. 2000, MNRAS, 314, 858

Lucas, P. W., Roche, P. F., Allard, F., \& Hauschildt, P. H. 2001, MNRAS, 326, 695

Luhman, K. L., Liebert, J., \& Rieke, G. H. 1997, ApJ, 489, L165

Luhman, K. L. 1999, ApJ, 525, 466

Luyten, W. J. 1979, LHS catalogue. A catalogue of stars with proper motions exceeding $0^{\prime \prime} 5$ annually, 2nd ed. (Minneapolis: University of Minnesota)

Martín, E. L., Rebolo, R., \& Zapatero Osorio, M. R. 1996, ApJ, 469, 706

Martín, E. L., Delfosse, X., Basri, G., et al. 1999, AJ, 118, 2466

Martín, E. L., Dougados, C., Magnier, E., et al. 2001, ApJ, 561, L195

Mizuno, A., Hayakawa, T., Tachihara, K., et al. 1999, PASJ, 51, 859

Mokler, F., \& Stelzer, B. 2002, A\&A, 391, 1025

Moraux, E., Bouvier, J., Stauffer, J. R., \& Cuillandre, J.-C. 2003, A\&A, 400, 891

Morrison, J. E., Röser, S., McLean, B., et al. 2001, AJ, 121, 1752

Natta, A., \& Testi, L. 2001, A\&A, 376, L22

Neuhäuser, R., \& Comerón, F. 1998, Science, 282, 83

Neuhäuser, R., \& Comerón, F. 1999, A\&A, 350, 612

Neuhäuser, R., Brandner, W., Alves, J., et al. 2002, A\&A, 384, 999

Oasa, Y., Tamura, M., \& Sugitani, K. 1999, ApJ, 526, 336

Oliveira, J. M., Jeffries, R. D., Kenyon, M. J., et al. 2002, A\&A, 382, L22
Oliveira, J. M., Jeffries, R. D., \& van Loon, J. Th. 2003 [astro-ph/0310254]

Persi, P., Marenzi, A. R., Olofsson, G., et al. 2000, A\&A, 357, 219

Pickett, B. K., Durisen, R. H., Cassen, P., \& Mejia, A. C. 2000, ApJ, 540, L95

Prusti, T., Clark, F. O., Whittet, D. C. B., et al. 1991, MNRAS, 251, 303

Reid, I. N., \& Hawley, S. L. 2000, New Light on Dark Stars (SpringerPraxis)

Reipurth, B., \& Clarke, C. 2001, AJ, 122, 432

Reipurth, B., \& Zinnecker, H. 1993, A\&A, 278, 81

Rieke, G. K., \& Lebofsky, M. J. 1985, ApJ, 288, 618

Robin, A., \& Crezé, M. 1986, A\&A, 157, 71

Schwartz, R. D. 1977, ApJS, 35, 161

Schwartz, R. D. 1991, in Low Mass Star Formation in Southern Molecular Clouds, ed. B. Reipurth, ESO Scientific Report No.11, 93

Stetson, P. B. 1987, PASP, 99, 191

Sterzik, M. F., \& Durisen, R. H. 1999, in Proc. Star Formation, ed. T. Nakamoto, 387

Tej, A., Sahu, K. C., Chandrasekhar, T., \& Ashok, N. M. 2002, ApJ, 578,523

Whittet, D. C. B., Prusti, T., Franco, G. A. P., et al. 1997, A\&A, 327, 1194

Wilking, B. A., Greene, T. P., \& Meyer, M. R. 1999, AJ, 117, 469

Zapatero Osorio, M. R., Martín, E. L., \& Rebolo, R. 1997, A\&A, 323, 105

Zapatero Osorio, M. R., Béjar, Martín, E. L., et al. 2002a, ApJ, 569, L99

Zapatero Osorio, M. R., Béjar, V. J. S., Pavlenko, Y., et al. 2002b, A\&A, 384, 937 